TRANSACTIONS OF THE

AMERICAN MATHEMATICAL SOCIETY

Volume 357, Number 11, Pages 4445-4479

S 0002-9947(05)03965-6

Article electronically published on June 10, 2005

\title{
RIEMANNIAN NILMANIFOLDS AND THE TRACE FORMULA
}

\author{
RUTH GORNET
}

\begin{abstract}
This paper examines the clean intersection hypothesis required for the expression of the wave invariants, computed from the asymptotic expansion of the classical wave trace by Duistermaat and Guillemin. The main result of this paper is the calculation of a necessary and sufficient condition for an arbitrary Riemannian two-step nilmanifold to satisfy the clean intersection hypothesis. This condition is stated in terms of metric Lie algebra data. We use the calculation to show that generic two-step nilmanifolds satisfy the clean intersection hypothesis. In contrast, we also show that the family of two-step nilmanifolds that fail the clean intersection hypothesis are dense in the family of two-step nilmanifolds. Finally, we give examples of nilmanifolds that fail the clean intersection hypothesis.
\end{abstract}

\section{INTRODUCTION}

The spectrum of a closed Riemannian manifold $(M, g)$, denoted $\operatorname{spec}(M, g)$, is the collection of eigenvalues of the Laplace-Beltrami operator $\Delta$ acting on smooth functions. Two manifolds $(M, g)$ and $\left(M^{\prime}, g^{\prime}\right)$ are isospectral if $\operatorname{spec}(M, g)=\operatorname{spec}\left(M^{\prime}, g^{\prime}\right)$. The length spectrum of $(M, g)$, denoted by $\operatorname{spec}_{[L]}(M, g)$, is the collection of lengths of smoothly closed geodesics of $(M, g)$, counted with multiplicity. The multiplicity of a length is defined as the number of free homotopy classes of loops containing a closed geodesic of that length. The absolute length spectrum of a Riemannian manifold $(M, g)$, denoted $\operatorname{spec}_{L}(M, g)$, is the set of lengths of smoothly closed geodesics, with no multiplicity assigned.

A major open question in spectral geometry is the precise relation between the Laplace spectrum on functions and the (absolute) length spectrum. In a few cases, the Laplace spectrum on functions and the length spectrum are known to be equivalent. Huber showed that two closed Riemann surfaces are isospectral if and only if they have the same length spectrum (see $[\mathrm{Bu}]$ for an exposition and references), and the Poisson summation formula shows that two flat tori are isospectral if and only if they have the same length spectrum (see [BGM]). In contrast, the Zöll and standard spheres provide us with examples of manifolds that have the same length spectrum, but not the same Laplace spectrum. Indeed, by definition, all of the closed geodesics on Zöll spheres have length an integer multiple of $2 \pi$; the same is

Received by the editors August 28, 2003.

2000 Mathematics Subject Classification. Primary 35P20, 58J53, 53C22; Secondary 58J40, $22 \mathrm{E} 25$.

Key words and phrases. Isospectral manifolds, nilmanifolds, length spectrum, trace formula.

This material is based in part on work supported by the Texas Advanced Research Program under Grant No. 003644-002 and by NSF grants DMS-9753220 (preliminary) and DMS-0204648.

(C)2005 American Mathematical Society Reverts to public domain 28 years from publication 
true for standard spheres. However, the standard sphere is known to be spectrally determined in dimension less than or equal to six. (See [Bs] and [BGM].)

Using the heat equation, Colin de Verdière $[\mathrm{CdV}]$ has shown that generically (in the family of all Riemannian manifolds), the Laplace spectrum determines the absolute length spectrum. This result supports the widely held belief that all isospectral manifolds must share the same absolute length spectrum.

In sharp contrast, Miatello and Rossetti $[\mathrm{MR}$ have constructed pairs of compact flat manifolds that are isospectral on one-forms but which do not have the same absolute length spectrum. Also in contrast, C. S. Gordon G1 has constructed pairs of isospectral Heisenberg manifolds that have unequal multiplicities in the length spectrum, and Gornet Gt1 has constructed other higher-step nilmanifolds with this property. There is no known example of a pair of manifolds that are isospectral on functions but with unequal absolute length spectra.

Our objects of study in this paper are Riemannian two-step nilmanifolds: closed manifolds of the form $(\Gamma \backslash G, g)$, where $G$ is a simply-connected two-step nilpotent Lie group, $\Gamma$ is a uniform (i.e., $\Gamma \backslash G$ compact) discrete subgroup of $G$, and $g$ is a left invariant metric on $G$, which descends to a Riemannian metric on $\Gamma \backslash G$ that we also denote by $g$. A Heisenberg manifold is a two-step Riemannian nilmanifold whose covering Lie group $G$ is one of the $(2 n+1)$-dimensional Heisenberg Lie groups. Twostep nilmanifolds in general, and Heisenberg manifolds in particular, have been a rich source of examples of isospectral manifolds, producing a wealth of geometric properties not determined by the spectrum; see [DG1], [DG2], [0], [P1], [P2], [P3], [P4], GW1, GW2, GW3, G1, G2, G3], E1].

The classical way to express the relation between the Laplace spectrum and the length spectrum on general manifolds is the following. For $(M, g)$ a Riemannian manifold, define $e_{M}(t)=\operatorname{trace}(\exp (i t \sqrt{\Delta}))=\sum_{\lambda \in \operatorname{spec}(M, g)} e^{i t \sqrt{\lambda}}$. This is a spectrally determined tempered distribution of $t$. The classical trace formula, arising from the study of the wave equation, provides information about the singularities of $e_{M}(t)$. In particular [DGu,

(1) the singular support of $e_{M}(t)$ is contained in $\operatorname{spec}_{L}(M, g)$, and

(2) if $\tau$ is in $\operatorname{spec}_{L}(M, g)$ and $\tau$ satisfies a Clean Intersection Hypothesis, then $e_{M}(t)$ is a classical conormal distribution in a neighborhood of $\tau$, and the singularities of $e_{M}(t)$ at $\tau$ provide geometric information about $(M, g)$, the wave invariants.

See [St] for an excellent introduction to the basics of distribution theory in general and tempered distributions in particular. See DGu for more information about the classical trace formula.

Note that $e_{M}(t)$ is spectrally determined; i.e., if $(M, g)$ and $\left(M^{\prime}, g^{\prime}\right)$ are isospectral, then $e_{M}(t)=e_{M^{\prime}}(t)$. Recently S. Zelditch [Z1], [Z2] has used the wave invariants to construct plane domains that are spectrally determined within the family of plane domains with analytic boundary. (See also $[\mathrm{Gu}]$.)

The fact that the singular support of $e_{M}(t)$ is contained in $\operatorname{spec}_{L}(M, g)$ is physically quite natural. One expects that sound waves propagate along geodesics, hence singularities should occur when these sound waves intersect themselves, i.e., meet up while travelling along smoothly closed geodesics.

The generic results of Colin de Verdière [CdV], described above, can also be obtained from the classical trace formula $[\overline{D G u}$. In the case of compact, hyperbolic 
manifolds, this arises from the Selberg Trace Formula [Sel]. (See also [Ch, Chapter $\mathrm{XI}]$.)

The genericity hypotheses of Colin de Verdière and Duistermaat-Guillemin require that all closed geodesics be isolated and that lengths be multiplicity free, i.e., a length $\tau \in \operatorname{spec}_{L}(M, g)$ appears in exactly one free homotopy class (and its inverse). Nilmanifolds possess a great deal of symmetry so that closed geodesics always come in large-dimensional families, thus failing these generic hypotheses. Consequently, generic results relating the Laplace and length spectra say nothing about the many known examples of isospectral nilmanifolds and other isospectral families of manifolds.

A distinct issue from genericity, and the focus of this paper, is that of the Clean Intersection Hypothesis, which is defined in Definition 1.4 below. Roughly speaking, the Clean Intersection Hypothesis requires that fixed point sets of the differential of the geodesic flow at initial velocities producing smoothly closed geodesics behave nicely. The geometric expression of the wave invariants given by Duistermaat and Guillemin is valid when the Clean Intersection Hypothesis is satisfied.

The main theorem (see Theorem 2.6 and Theorem 3.1) of this paper is the calculation of necessary and sufficient conditions for a two-step nilmanifold to satisfy the Clean Intersection Hypothesis. This condition is expressed entirely in terms of metric Lie algebra data. See (1.9) for an example of a (Heisenberg) two-step nilmanifold that fails the Clean Intersection Hypothesis.

A corollary to this condition is (see Corollary 2.9 and Theorem 2.10):

Theorem. The family of two-step nilmanifolds that fail the Clean Intersection Hypothesis are dense in the family of two-step nilmanifolds. Generic two-step nilmanifolds satisfy the Clean Intersection Hypothesis.

The existence of "unclean" left invariant metrics on Heisenberg manifolds was previously observed by H. Pesce in [P4, Remark II.5].

This paper is organized so that anyone with a background in Riemannian geometry should be able to read Section 1 and Section 2, including the statements of the main theorem and its corollaries. The highly technical aspects of two-step nilmanifolds and the proof of main theorems are left to Section 3. Section 1 starts with an exposition of the Clean Intersection Hypothesis, and directly calculates the Clean Intersection Hypothesis on all flat tori (Example 1.19) and for certain three-dimensional Heisenberg manifolds (Example 1.20). Section 2 includes a very brief, expository introduction to two-step nilmanifolds, which is enough to state the main theorem (Theorem 2.6) and its corollaries. In contrast to its statement, the proof of the main theorem is quite technical and is the focus of Section 3, which also includes detailed background information on the length spectrum of two-step nilmanifolds.

The author wishes to thank Alejandro Uribe and Jeffrey M. Lee for inspiring conversations about this paper.

\section{The Clean Intersection Hypothesis}

Throughout this paper, a prime (e.g. $\left.X^{\prime}(t)\right)$ will denote derivation with respect to the $t$ or $r$ variable, and a dot (e.g. $\dot{\alpha}(s))$ will denote derivation with respect to the $s$ variable. 
1.1 Notation. Denote by $\mathfrak{X}$ the unit tangent bundle of a Riemannian manifold $(M, g)$. For $v_{p} \in \mathfrak{X}$, denote by $\sigma_{v_{p}}(t)$ the unique geodesic in $(M, g)$ with initial velocity $v_{p}$. So $\sigma_{v_{p}}(0)=p \in M$, and $\sigma_{v_{p}}^{\prime}(0)=v_{p} \in \mathfrak{X}$. Denote geodesic flow on $(M, g)$ by $\Phi: \mathbb{R} \times \mathfrak{X} \rightarrow \mathfrak{X}$. So

$$
\Phi\left(t, v_{p}\right)=\sigma_{v_{p}}^{\prime}(t) .
$$

Denote by $c$ a free homotopy class of loops of $M$. For $\tau>0$, define

$$
F(\tau, c)=\left\{v_{p} \in \mathfrak{X}: \Phi\left(\tau, v_{p}\right)=v_{p}, \sigma_{\left.v_{p}\right|_{[0, \tau]}} \in c\right\} .
$$

$F(\tau, c)$ is the set of all initial velocities producing smoothly closed, unit speed geodesics of period $\tau$ (i.e. of length $\tau$ ) that are, as closed loops, contained in the free homotopy class $c$. Note that $F(\tau, c)$ is empty if there does not exist a closed, unit speed geodesic in the free homotopy class $c$ of length $\tau$.

The calculation of the wave invariants by Duistermaat and Guillemin uses the cotangent bundle $[\overline{\mathrm{DGu}}]$. As we are working on Riemannian manifolds, there is a natural identification between the cotangent bundle and the tangent bundle in this context. The paper $\mathrm{GuU}$ has a nice expository explanation of the wave trace, and the set $F(\tau, c)$ here is equal to the set $W_{\tau}(c)$ there.

For $\tau>0$, define $\Phi_{\tau}: \mathfrak{X} \rightarrow \mathfrak{X}$ by

$$
\Phi_{\tau}\left(v_{p}\right)=\Phi\left(\tau, v_{p}\right) .
$$

If $v_{p} \in F(\tau, c)$, then $\Phi_{\tau}\left(v_{p}\right)=v_{p}$, so

$$
\text { if } v_{p} \in F(\tau, c) \text {, then } \Phi_{\tau_{*_{v}}}: T_{v_{p}} \mathfrak{X} \rightarrow T_{v_{p}} \mathfrak{X} \text {. }
$$

Define $\operatorname{FPS}\left(\tau, v_{p}\right)$ to be the fixed point set of $\Phi_{\tau *_{v_{p}}}$.

1.4 Definition. A period $\tau$ in $\operatorname{spec}_{L}(M, g)$ satisfies the Clean Intersection Hypothesis if for all $c$ such that $F(\tau, c)$ is nonempty, $F(\tau, c)$ is a finite union of submanifolds of $\mathfrak{X}$, and for all $v_{p}$ in $F(\tau, c), T_{v_{p}} F(\tau, c)$ equals the fixed point set of $\Phi_{\tau_{*_{v_{p}}}}$; that is, if $v_{p} \in F(\tau, c)$,

$$
T_{v_{p}} F(\tau, c)=\operatorname{FPS}\left(\tau, v_{p}\right)
$$

The manifold $(M, g)$ satisfies the Clean Intersection Hypothesis if for all $\tau$ in $\operatorname{spec}_{L}(M, g), \tau$ satisfies the Clean Intersection Hypothesis.

1.5 Remark. Since $\Phi_{\left.\tau\right|_{F(\tau, c)}}=\operatorname{Id}_{F(\tau, c)}$, if $v_{p} \in F(\tau, c)$, then

$$
T_{v_{p}} F(\tau, c) \subset \operatorname{FPS}\left(\tau, v_{p}\right) \text {. }
$$

The issue, then, when studying the Clean Intersection Hypothesis is whether there can exist fixed points of $\Phi_{\tau *_{v_{p}}}$ that are normal to $F(\tau, c)$.

The manifolds we consider in this paper are constructed as follows. Let $G$ be a simply-connected Lie group, and consider the action of $G$ on itself by left translations. Let $\Gamma$ be a cocompact (i.e., $\Gamma \backslash G$ compact), discrete subgroup of $G$; the existence of $\Gamma$ implies that $G$ is unimodular. A Riemannian metric $g$ is left invariant if the left translations $L_{p}$ of $G$ are isometries for all $p$ in $G$, so the action of $\Gamma$ on $G$ is by isometries. Note that a left invariant metric on $G$ is determined by specifying an inner product $\langle$,$\rangle on the Lie algebra \mathfrak{g}$ of $G$ and vice versa. A Lie algebra together with an inner product $(\mathfrak{g},\langle\rangle$,$) is called a metric Lie algebra. The$ 
left invariant metric $g$ descends to a Riemannian metric on $\Gamma \backslash G$, also denoted by $g$. With this metric, the mapping

$$
\pi_{1}:(G, g) \rightarrow(\Gamma \backslash G, g)
$$

is a Riemannian covering.

As is standard, we study closed geodesics on $(\Gamma \backslash G, g)$ by lifting them to the universal cover $(G, g)$.

1.7 Definition. Let $\sigma$ be a unit speed geodesic of $(G, g)$. A nonidentity element $\gamma$ in $G$ translates $\sigma$ by an amount $\tau>0$ if $\gamma \sigma(t)=\sigma(t+\tau)$ for all $t \in \mathbb{R}$. The number $\tau$ is called a period of $\gamma$.

As $G$ is simply-connected, the free homotopy classes of $\Gamma \backslash G$ correspond to the conjugacy classes in the fundamental group $\Gamma$. We denote the conjugacy class in $\Gamma$ containing $\gamma \in \Gamma$ by $c_{\gamma}$, so $c_{\gamma}=\left\{\gamma^{\prime} \gamma \gamma^{\prime-1}: \gamma^{\prime} \in \Gamma\right\}$. As $\pi_{1}$ is a Riemannian covering, we have the following:

1.8 Property. The manifold $(\Gamma \backslash G, g)$ contains a closed geodesic of length $\tau$ in the free homotopy class $c_{\gamma}$ if and only if the manifold $(G, g)$ contains a unit speed geodesic $\sigma(t)$ and there exists $\gamma^{\prime} \in c_{\gamma}$ such that $\gamma^{\prime}$ translates $\sigma$ with period $\tau$. Under the mapping $\pi_{1}$, the geodesic $\sigma$ then projects to a smoothly closed geodesic of length $\tau$ on $(\Gamma \backslash G, g)$ in the free homotopy class $c_{\gamma}$.

We likewise study the geodesic flow of $(\Gamma \backslash G, g)$ by first studying the geodesic flow of $(G, g)$. Let $\mathfrak{X}$ (resp. $\tilde{\mathfrak{X}}$ ) denote the unit tangent bundle of $\Gamma \backslash G$ (resp. $G$ ).

Because the metric on $G$ is left invariant, the unit tangent bundle $\tilde{\mathfrak{X}}$ is equivalent to the sphere bundle (viewing $\mathfrak{g}$ as $T_{e} G$ )

$$
\tilde{\mathfrak{X}} \cong G \times S^{n-1}=\{(\tilde{p}, v): \tilde{p} \in G, v \in \mathfrak{g},|v|=1\},
$$

under the mapping $v_{\tilde{p}} \mapsto\left(\tilde{p}, L_{\tilde{p}^{-1} *_{e}} v\right)$. We use $v_{\tilde{p}} \in \tilde{\mathfrak{X}}$ and $(\tilde{p}, v) \in G \times S^{n-1}$ interchangeably in what follows. Note that the left action of $G$ on $G$ by isometries determines an action of $G$ on $\tilde{\mathfrak{X}}$ by isometries:

$$
\tilde{q} \cdot v_{\tilde{p}}=\tilde{q} \cdot(\tilde{p}, v)=\left(L_{\tilde{q}}(\tilde{p}), v\right)=v_{\tilde{q} \tilde{p}}
$$

The subgroup $\Gamma$ then acts on $\tilde{\mathfrak{X}}$ by isometries as above, and

$$
\mathfrak{X} \cong \Gamma \backslash \tilde{\mathfrak{X}} \cong(\Gamma \backslash G) \times S^{n-1} .
$$

We let $\pi$ denote the canonical mapping

$$
\pi: \tilde{\mathfrak{X}} \rightarrow \Gamma \backslash \tilde{\mathfrak{X}},
$$

so that $\pi_{1}$ defined in (1.6) is just $\pi$ restricted to the first factor.

Throughout this paper, elements with tildes such as $\tilde{p}, v_{\tilde{p}}$, denote elements in $G$ and $\tilde{\mathfrak{X}}$, respectively; elements without tildes such as $p$ and $v_{p}$ denote the image of $\tilde{p}$ and $v_{\tilde{p}}$ in $\Gamma \backslash G$ and $\mathfrak{X}$, respectively, under the canonical mapping $\pi: \tilde{\mathfrak{X}} \rightarrow \mathfrak{X}$. The element $e$ is the identity element of $G$ and we denote $\bar{e}=\pi_{1}(e)$.

We define

$$
\tilde{F}(\tau, \gamma)=\left\{v_{\tilde{p}} \in \tilde{\mathfrak{X}}: \tilde{\Phi}_{\tau}\left(v_{\tilde{p}}\right)=\gamma \cdot v_{\tilde{p}}\right\}
$$


With these identifications, we have, for the free homotopy class $c_{\gamma}$ and the length $\tau>0$ in the manifold $\Gamma \backslash G$,

$$
\begin{aligned}
F\left(\tau, c_{\gamma}\right) & =\left\{v_{p} \in \mathfrak{X}: \Phi_{\tau}\left(v_{p}\right)=v_{p}, \sigma_{\left.v_{p}\right|_{[0, \tau]}} \in c_{\gamma}\right\} \\
& =\left\{\pi\left(v_{\tilde{p}}\right): v_{\tilde{p}} \in \tilde{\mathfrak{X}}, \exists \gamma^{\prime} \in c_{\gamma}, \sigma_{v_{\tilde{p}}}(t+\tau)=\gamma^{\prime} \sigma_{v_{\tilde{p}}}(t) \forall t\right\} \\
& =\left\{\pi\left(v_{\tilde{p}}\right): v_{\tilde{p}} \in \tilde{\mathfrak{X}}, \exists \gamma^{\prime} \in c_{\gamma}, \tilde{\Phi}_{\tau}\left(v_{\tilde{p}}\right)=\gamma^{\prime} \cdot v_{\tilde{p}}\right\} \\
& =\left\{\pi\left(\tilde{F}\left(\tau, \gamma^{\prime}\right)\right): \gamma^{\prime} \in c_{\gamma}\right\} \\
& =\pi(\tilde{F}(\tau, \gamma)) .
\end{aligned}
$$

To see the last equality, note that if $\gamma^{\prime} \in c_{\gamma}$, then there exists $\gamma^{\prime \prime} \in \Gamma$ such that $\gamma=\gamma^{\prime \prime} \gamma^{\prime} \gamma^{\prime \prime}-1$, i.e., $\gamma^{\prime \prime} \gamma^{\prime}=\gamma \gamma^{\prime \prime}$. If $v_{\tilde{p}} \in \tilde{\mathfrak{X}}$ such that $\tilde{\Phi}_{\tau}\left(v_{\tilde{p}}\right)=\gamma^{\prime} \cdot v_{\tilde{p}}$, then $\sigma_{v_{\tilde{p}}}(t+\tau)=\gamma^{\prime} \sigma_{v_{\tilde{p}}}(t)$ for all $t \in \mathbb{R}$, so

$$
\gamma^{\prime \prime} \sigma_{v_{\tilde{p}}}(t+\tau)=\gamma^{\prime \prime} \gamma^{\prime} \sigma_{v_{\tilde{p}}}(t)=\gamma \gamma^{\prime \prime} \sigma_{v_{\tilde{p}}}(t)
$$

Since left translations are isometries, this implies

$$
\sigma_{\gamma^{\prime \prime} \cdot v_{\tilde{p}}}(t+\tau)=\gamma \sigma_{\gamma^{\prime \prime} \cdot v_{\tilde{p}}}(t),
$$

i.e., $\tilde{\Phi}_{\tau}\left(\gamma^{\prime \prime} \cdot v_{\tilde{p}}\right)=\tilde{\Phi}_{\tau}\left(v_{\gamma^{\prime \prime} \cdot \tilde{p}}\right)=\gamma \cdot v_{\gamma^{\prime \prime} \cdot \tilde{p}}$. This implies that $\tilde{F}\left(\tau, \gamma^{\prime}\right)=\gamma^{\prime \prime} \tilde{F}(\tau, \gamma)$, which implies $\pi\left(\tilde{F}\left(\tau, \gamma^{\prime}\right)\right)=\pi(\tilde{F}(\tau, \gamma))$. We have shown:

1.15 Proposition. With notation as above, let $v_{p} \in \mathfrak{X}$. The vector $v_{p} \in F\left(\tau, c_{\gamma}\right)$ if and only if there exists $v_{\tilde{p}} \in \pi^{-1}\left(v_{p}\right)$ such that $\tilde{\Phi}_{\tau}\left(v_{\tilde{p}}\right)=\gamma \cdot v_{\tilde{p}}$. In this case,

$$
\tilde{\Phi}_{\tau *_{\tilde{p}}}: T_{v_{\tilde{p}}} \tilde{\mathfrak{X}} \rightarrow T_{\gamma \cdot v_{\tilde{p}}} \tilde{\mathfrak{X}} \text {. }
$$

As $\tilde{\mathfrak{X}}$ is a Riemannian cover of $\mathfrak{X}$, we study fixed points of $\Phi_{\tau *_{v_{p}}}$ by studying $\tilde{\Phi}_{\tau *_{\tilde{p}}}: T_{v_{\tilde{p}}} \tilde{\mathfrak{X}} \rightarrow T_{\gamma \cdot v_{\tilde{p}}} \tilde{\mathfrak{X}}$ for $v_{\tilde{p}} \in \pi^{-1}\left(v_{p}\right)$.

Note that under the identification $\tilde{\mathfrak{X}} \cong G \times S^{n-1}$, we may write $T_{v_{\tilde{p}}} \tilde{\mathfrak{X}}$ as follows:

$$
T_{v_{\tilde{p}}} \tilde{\mathfrak{X}}=T_{\tilde{p}} G \times T_{v} S^{n-1}=\left\{\left(u, v^{\perp}\right): u \in T_{\tilde{p}} G, v^{\perp} \in \mathfrak{g}, v^{\perp} \perp v\right\},
$$

where by $S^{n-1}$ we mean the unit sphere in $\mathfrak{g}$. Likewise we have

$$
T_{v_{p}} \mathfrak{X}=T_{p}(\Gamma \backslash G) \times T_{v} S^{n-1}=\left\{\left(u, v^{\perp}\right): u \in T_{p}(\Gamma \backslash G), v^{\perp} \in \mathfrak{g}, v^{\perp} \perp v\right\} .
$$

We abuse notation slightly by identifying the components of

$$
\pi_{*_{\tilde{p}}}\left(\left(u, v^{\perp}\right)_{v_{\tilde{p}}}\right)=\left(u, v^{\perp}\right)_{v_{p}}
$$

and

$$
\left(u, v^{\perp}\right)_{v_{\tilde{p}}}
$$

We may do this since $\pi$ is a Riemannian covering, so $T_{p}(\Gamma \backslash G)$ and $T_{\tilde{p}} G$ are isometric. Since $\gamma \cdot v_{\tilde{p}}=v_{\gamma \cdot \tilde{p}}$,

$$
T_{\gamma \cdot v_{\tilde{p}}} \tilde{\mathfrak{X}}=T_{\gamma \cdot \tilde{p}} G \times T_{v} S^{n-1} .
$$

We may use the left action on $G$ to identify elements of $T_{\tilde{p}} G$ and $T_{\gamma \cdot \tilde{p}} G$, i.e., to identify $T_{v_{\tilde{p}}} \tilde{\mathfrak{X}}$ and $T_{\gamma \cdot v_{\tilde{p}}} \tilde{\mathfrak{X}}$. We have shown:

1.18 Proposition. With notation as above, let $v_{p} \in F\left(\tau, c_{\gamma}\right)$. An element $\left(u, v^{\perp}\right)_{v_{p}}$ $\in T_{v_{p}} \mathfrak{X}$ is a fixed point of $\Phi_{\tau_{*_{v_{p}}}}$ if and only if there exists $v_{\tilde{p}} \in \pi^{-1}\left(v_{p}\right)$ such that

$$
\tilde{\Phi}_{\tau *_{v_{\tilde{p}}}}\left(\left(u, v^{\perp}\right)_{v_{\tilde{p}}}\right)=\left(u, v^{\perp}\right)_{\gamma \cdot v_{\tilde{p}}} .
$$


Two examples. The Clean Intersection Hypothesis is difficult to grasp intuitively, and as it is the focus of this paper, we present the following two "warm-up" examples before stating and proving the more general results in Section 2 and Section 3.

The purpose of looking at these particular examples is to illustrate the process on manifolds that are familiar and interesting to the general audience.

1.19 Flat torus. We show that any flat torus satisfies the Clean Intersection Hypothesis.

Let $G=\mathbb{R}^{n}$. Here $\Gamma=L$, a lattice of full rank in $\mathbb{R}^{n}$. Then $L \backslash \mathbb{R}^{n}$ with the metric induced from the Euclidean metric on $\mathbb{R}^{n}$ is a closed Riemannian manifold. Note that both $L \backslash \mathbb{R}^{n}$ and $\mathbb{R}^{n}$ are Lie groups under addition and are endowed with a left (and right) invariant metric, as translations are isometries. As in the general case, we identify

$$
\tilde{\mathfrak{X}} \cong \mathbb{R}^{n} \times S^{n-1}=\left\{(\tilde{p}, v): \tilde{p} \in \mathbb{R}^{n}, v \in \mathbb{R}^{n},|v|=1\right\} .
$$

We continue to use $v_{\tilde{p}}$ and $(\tilde{p}, v)$ interchangeably and $v_{p}$ and $(p, v)$ interchangeably.

Geodesics in $\mathbb{R}^{n}$ are just straight lines, so geodesics in $L \backslash \mathbb{R}^{n}$ are projections of straight lines. For $v_{\tilde{p}} \in \tilde{\mathfrak{X}}, \sigma_{v_{\tilde{p}}}(t)=\tilde{p}+t v \in \mathbb{R}^{n}$ and $\tilde{\Phi}\left(t, v_{\tilde{p}}\right)=v_{\tilde{p}+t v}=(\tilde{p}+t v, v)$. Since $\pi_{1}: \mathbb{R}^{n} \rightarrow L \backslash \mathbb{R}^{n}$ is a Riemannian submersion with totally geodesic fibers, for $v_{p} \in \mathfrak{X}, \Phi\left(t, v_{p}\right)=v_{p+t v}$.

Free homotopy classes of $L \backslash \mathbb{R}^{n}$ correspond to conjugacy classes in $L$. As $\mathbb{R}^{n}$ is abelian, the free homotopy classes of $L \backslash \mathbb{R}^{n}$ are in one-to-one correspondence with the elements of $L$. The unit speed geodesic $\sigma_{v_{\tilde{p}}}(t)$ on $\mathbb{R}^{n}$ projects to a closed geodesic of $L \backslash \mathbb{R}^{n}$ with period $\tau$ in the free homotopy class $c_{l}$ if and only if $\sigma_{v_{\tilde{p}}}(t+\tau)=$ $l+\sigma_{v_{\tilde{p}}}(t)$ for all $t$. In this case

$$
\sigma_{v_{\tilde{p}}}(t+\tau)=\tilde{p}+(t+\tau) v=l+\sigma_{v_{\tilde{p}}}(t)=l+\tilde{p}+t v,
$$

so $l=v \tau$ and, as $v$ is a unit vector, $\tau=|l|$. The length spectrum of $L \backslash \mathbb{R}^{n}$ is thus

$$
\operatorname{spec}_{L}\left(L \backslash \mathbb{R}^{n}\right)=\{|l|: l \in L\} .
$$

As in (1.13), $F\left(\tau, c_{l}\right)=\pi(\tilde{F}(\tau, l))$, and

$$
\begin{aligned}
\tilde{F}(\tau, l) & =\left\{v_{\tilde{p}}: v_{\tilde{p}} \in \tilde{\mathfrak{X}}, \tilde{\Phi}_{\tau}\left(v_{\tilde{p}}\right)=l \cdot v_{\tilde{p}}\right\} \\
& =\left\{v_{\tilde{p}}: v_{\tilde{p}} \in \tilde{\mathfrak{X}}, \tau v=l\right\} \\
& =\mathbb{R}^{n} \times\{l /|l|\} \quad \text { if }|l|=\tau .
\end{aligned}
$$

We conclude that if $|l| \neq \tau$, then $F\left(\tau, c_{l}\right)=\emptyset$, and if $|l|=\tau$, then

$$
F\left(\tau, c_{l}\right)=L \backslash \mathbb{R}^{n} \times\{l /|l|\} .
$$

Clearly, $F\left(\tau, c_{l}\right)$ is diffeomorphic to $L \backslash \mathbb{R}^{n}$.

If $v_{p} \in F\left(|l|, c_{l}\right)$, by (1.17) and Remark 1.5,

$$
T_{v_{p}} F\left(|l|, c_{l}\right)=T_{p}\left(L \backslash \mathbb{R}^{n}\right) \times\{0\} \subset \operatorname{FPS}\left(|l|, v_{p}\right) .
$$

Thus, for $(u, 0)_{v_{p}} \in T_{v_{p}} F\left(|l|, c_{l}\right), \Phi_{\tau *_{v_{p}}}\left((u, 0)_{v_{p}}\right)=(u, 0)_{v_{p}}$. An arbitrary element of $T_{v_{p}} \mathfrak{X}$ that is normal to $T_{v_{p}} F\left(|l|, c_{l}\right)$ is given by $\left(0, v^{\perp}\right)_{v_{p}}$, where $v^{\perp}$ is any vector in $\mathbb{R}^{n}$ (viewed here as $\mathbb{R}^{n}=T_{e} \mathbb{R}^{n}$ ) that is orthogonal to $v$. (See Remark 1.5.)

We show that $\left(0, v^{\perp}\right)_{v_{p}}$ cannot be a fixed point of $\Phi_{\tau *_{v_{p}}}$ by showing that

$$
\Phi_{\tau *_{v_{p}}}\left(\left(0, v^{\perp}\right)\right)=\left(\tau v^{\perp}, v^{\perp}\right)
$$

thus vectors normal to $F\left(|l|, c_{l}\right)$ cannot be fixed points. 
Let $\alpha(s)=\left(p, \cos (s) v+\sin (s) v^{\perp}\right) \in \mathfrak{X}$. Then $\alpha(0)=(p, v)$, and $\dot{\alpha}(0)=\left(0, v^{\perp}\right) \in$ $T_{v_{p}} \mathfrak{X}$. Also $\Phi_{\tau}(\alpha(0))=(p+\tau v, v)$, and $\left.\frac{d}{d s}\right|_{0} \Phi_{\tau}(\alpha(s))=\left(\tau v^{\perp}, v^{\perp}\right) \in T_{v_{p+\tau v}} \mathfrak{X}$. But $p+\tau v=p+l=p$ in $L \backslash \mathbb{R}^{n}$, so

$$
\Phi_{\tau *_{v_{p}}}\left(\left(0, v^{\perp}\right)_{v_{p}}\right)=\left(\tau v^{\perp}, v^{\perp}\right)_{v_{p}} \in T_{v_{p}} \mathfrak{X} .
$$

Clearly, the fixed point set of $\Phi_{\tau *_{v_{p}}}$ is $T_{v_{p}} F\left(\tau, c_{l}\right)$, and $L \backslash \mathbb{R}^{n}$ satisfies the Clean Intersection Hypothesis.

1.20 Three-dimensional Heisenberg manifold. We show that certain cocompact, discrete subgroups of the three-dimensional Heisenberg group produce manifolds that fail the Clean Intersection Hypothesis.

In this example, $G=H$, the three-dimensional Heisenberg group. Recall that the three-dimensional Heisenberg group can be defined by

$$
H=\left\{\left(\begin{array}{lll}
1 & x & z \\
0 & 1 & y \\
0 & 0 & 1
\end{array}\right): x, y, z \in \mathbb{R}\right\} .
$$

The mapping

$$
(x, y, z) \rightarrow\left(\begin{array}{ccc}
1 & x & z \\
0 & 1 & y \\
0 & 0 & 1
\end{array}\right)
$$

is a diffeomorphism from $\mathbb{R}^{3}$ to $H$ and determines local coordinates on $H$.

Note that other "standard" local coordinates are often used in the literature, which differ from those we use here by a diffeomorphism of $\mathbb{R}^{3}$. In particular, the logarithmic coordinates used in Section 3, in which we generalize the behavior of this example to all two-step nilmanifolds, uses a different coordinate system when applied to the Heisenberg group. The diffeomorphism from the matrix coordinate system used here to the logarithmic coordinate system used in Section 3 is $(x, y, z) \rightarrow \exp \left(x X+y Y+\left(z-\frac{1}{2} x y\right) Z\right)$.

In matrix coordinates, the corresponding Lie algebra $\mathfrak{h}$ of $H$ is determined in local coordinates at the point $\tilde{p}=(x, y, z)$ by the basis

$$
\begin{aligned}
X & =\frac{\partial}{\partial x}, \\
Y & =\frac{\partial}{\partial y}+x \frac{\partial}{\partial z}, \\
Z & =\frac{\partial}{\partial z} .
\end{aligned}
$$

Note that $[X, Y]=Z,[Y, X]=-Z$ and $Z$ spans the center $\mathfrak{z}$ of $\mathfrak{h}$. We specify a left invariant metric on $H$ by requiring that $\{X, Y, Z\}$ be an orthonormal basis of $\mathfrak{h}$.

Let $v=\bar{x} X+\bar{y} Y+\bar{z} Z$ be a unit vector at the identity $e=(0,0,0)$ of $H$. Kaplan Kp showed that (see also [G1, Prop. 2.7])

$$
\sigma_{v_{e}}(t)=\left(\begin{array}{ccc}
1 & x(t) & z(t) \\
0 & 1 & y(t) \\
0 & 0 & 1
\end{array}\right)
$$

where, if $\bar{z} \neq 0$,

$$
\left(\begin{array}{l}
x(t) \\
y(t)
\end{array}\right)=\frac{1}{\bar{z}}\left(\begin{array}{cc}
\sin (t \bar{z}) & \cos (t \bar{z})-1 \\
1-\cos (t \bar{z}) & \sin (t \bar{z})
\end{array}\right)\left(\begin{array}{l}
\bar{x} \\
\bar{y}
\end{array}\right)
$$


and

If $\bar{z}=0$, then

$$
z(t)=\left(\frac{1+\bar{z}^{2}}{2 \bar{z}}\right) t-\left(\frac{1-\bar{z}^{2}}{2 \bar{z}^{2}}\right) \sin (t \bar{z})+\frac{1}{2} x(t) y(t) .
$$

$$
x(t)=\bar{x} t, \quad y(t)=\bar{y} t, \quad z(t)=\frac{1}{2} x(t) y(t) .
$$

If $c(t)=(x(t), y(t), z(t))$ is a curve in $H$, then in the matrix coordinate system,

$$
\frac{d}{d t} c(t)=x^{\prime}(t) X+y^{\prime}(t) Y+\left(z^{\prime}(t)-x(t) y^{\prime}(t)\right) Z
$$

Thus

$$
\sigma_{v_{e}}^{\prime}(t)=x^{\prime}(t) X+y^{\prime}(t) Y+\bar{z} Z
$$

where $x(t)$ and $y(t)$ are given above.

Let $\Gamma$ be a cocompact, discrete subgroup of $H$. Let $\gamma \in \Gamma \cap Z(H)$, where $Z(H)$ denotes the center of $H$. So $\gamma=\left(0,0, z^{*}\right)$ for some $z^{*} \in \mathbb{R}$. C. S. Gordon G1 and later P. Eberlein [E1] computed the periods of $\gamma$, which are precisely

$$
\left\{\left|z^{*}\right| ; \sqrt{4 \pi k\left(\left|z^{*}\right|-\pi k\right)}, \quad 1 \leq k<\frac{\left|z^{*}\right|}{2 \pi}\right\} .
$$

1.21 Theorem. If $\left|z^{*}\right| \in 2 \pi \mathbb{Z}^{+}$, then the period $\tau=\left|z^{*}\right|$ fails the Clean Intersection Hypothesis.

An example of a cocompact, discrete subgroup $\Gamma$ that satisfies the hypothesis of this theorem is

$$
\Gamma=\{(2 \pi n, m, 2 \pi k): n, m, k \in \mathbb{Z}\} .
$$

Proof of Theorem 1.21. We omit many straightforward but tedious details, as this is just a special case of Theorem 2.6 below.

Since $\gamma \in Z(H)$ and $\tau=\left|z^{*}\right|$, careful analysis of the geodesic equations shows that

$$
\tilde{F}(\tau, \gamma)=H \times\{ \pm Z\}
$$

and

$$
F\left(\tau, c_{\gamma}\right)=\Gamma \backslash H \times\{ \pm Z\} .
$$

This also follows from Theorem 3.8 and Remarks 3.9 below. The sign of $\pm Z$ is determined by the sign of $z^{*}$. Thus, for $v_{\tilde{p}} \in \tilde{F}(\tau, \gamma)$,

$$
T_{v_{\tilde{p}}} \tilde{F}(\tau, \gamma)=\mathfrak{h} \times\{0\} .
$$

Without loss of generality, we assume that $z^{*}>0$. Now let

$$
\alpha(s)=(e, \sin (s) X+\cos (s) Z) \in \tilde{\mathfrak{X}} .
$$

Note that $\alpha(0)=Z_{e}$ and $\dot{\alpha}(0)=X_{Z_{e}}$. Plugging the initial velocity $v=\sin (s) X+$ $\cos (s) Z$ into the geodesic equations above, so that $\bar{x}=\sin (s), \bar{y}=0$, and $\bar{z}=\cos (s)$, we obtain

$$
\begin{aligned}
\tilde{\Phi}_{\tau}(\alpha(s))=(\tan s \sin (\tau \cos s), \tan s(1-\cos (\tau \cos s)), \\
\frac{1}{2}(\sec s+\cos s) \tau-\frac{1}{2} \tan ^{2} s \sin (2 \tau \cos s) ; \\
\sin s \cos (\tau \cos s), \sin s \sin (\tau \cos s), \cos s) .
\end{aligned}
$$


We compute that

$$
\frac{d}{d s} \tilde{\Phi}_{\tau}(\alpha(s))=(\sin \tau, 1-\cos \tau, 0 ; \cos \tau, \sin \tau, 0) .
$$

Since $\tau=\left|z^{*}\right| \in 2 \pi \mathbb{Z}^{+}$, we have shown that

$$
\tilde{\Phi}_{\tau * Z_{e}}\left((0, X)_{Z_{e}}\right)=(0, X)_{Z_{\gamma}},
$$

which by Proposition 1.18, shows that $(0, X)_{Z_{\bar{e}}}$ is a fixed point of $\Phi_{\tau * Z_{\bar{e}}}$, but clearly $(0, X)_{Z_{\bar{e}}}$ is not tangent to $F\left(\tau, c_{\gamma}\right)$.

\section{Two-step nilmanifolds and the Clean Intersection Hypothesis}

The purpose of this section is to state the main result of this paper: a necessary and sufficient condition for a two-step nilmanifold to satisfy the Clean Intersection Hypothesis. In this section, we also include several corollaries and their proofs. The proof of the main result, Theorem 2.6 (as generalized in Theorem 3.1) is very technical, and is the focus of Section 3.

Before proceeding, we need to introduce additional background information on two-step nilmanifolds. We continue the notation established in Section 1.

For a Lie algebra $\mathfrak{g}$, denote by $\mathfrak{g}^{(1)}$ the derived algebra $[\mathfrak{g}, \mathfrak{g}]$ of $\mathfrak{g}$. A Lie algebra $\mathfrak{g}$ is two-step nilpotent if $\mathfrak{g}^{(1)} \subset \mathfrak{z}$, where $\mathfrak{z}$ denotes the center of $\mathfrak{g}$, and $\mathfrak{g}^{(1)} \not \equiv 0$. A Lie group $G$ is two-step nilpotent if its Lie algebra is. Let $\Gamma$ be a cocompact, discrete subgroup of a simply-connected two-step nilpotent Lie group $G$ with left invariant metric $g$. The left invariant metric $g$ descends to a metric on $\Gamma \backslash G$ that we also denote by $g$. The locally homogeneous space $(\Gamma \backslash G, g)$ is called a Riemannian two-step nilmanifold.

As in Section 1, we study the geometry of $(\Gamma \backslash G, g)$ by studying the geometry of its simply-connected Riemannian covering $(G, g)$ and its associated metric Lie algebra $(\mathfrak{g},\langle\rangle$,$) .$

Throughout the remainder of this paper, $G$ will denote a simply-connected twostep nilpotent Lie group, $g$ will denote a left invariant metric on $G$, and $(\mathfrak{g},\langle\rangle$,$) will$ denote the associated metric Lie algebra. We denote the orthogonal complement of $\mathfrak{z}$ in $\mathfrak{g}$ by $\mathfrak{v}$ and write $\mathfrak{g}=\mathfrak{v} \oplus \mathfrak{z}$.

On all simply-connected nilpotent Lie groups, the Lie group exponential exp : $\mathfrak{g} \rightarrow G$ is a diffeomorphism $\left[\underline{\mathbb{R}}\right.$, so $G$ is diffeomorphic to $\mathbb{R}^{n}$, where $n=\operatorname{dim} \mathfrak{g}$. For two-step nilpotent Lie groups, by the Campbell-Baker-Hausdorff formula [V], we may easily write the group operation of $G$ in terms of the Lie algebra $\mathfrak{g}$ by

$$
\exp (X) \exp (Y)=\exp \left(X+Y+\frac{1}{2}[X, Y]\right),
$$

where $X, Y \in \mathfrak{g}$. Thus,

$$
\begin{aligned}
\exp (X)^{-1} & =\exp (-X), \\
\exp (X) \exp (Y) \exp (X)^{-1} & =\exp (Y+[X, Y]) \\
\exp (X) \exp (Y) \exp (X)^{-1} \exp (Y)^{-1} & =\exp ([X, Y]) .
\end{aligned}
$$

Let $\log : G \rightarrow \mathfrak{g}$ denote the inverse of the diffeomorphism exp .

We use the following information about two-step nilpotent metric Lie algebras, which was first developed by Kaplan $[\overline{K p}$ for the study of Heisenberg groups. 
2.2 Definition. Let $(\mathfrak{g},\langle\rangle$,$) be a two-step nilpotent metric Lie algebra, \mathfrak{g}=\mathfrak{v} \oplus \mathfrak{z}$. Define a linear transformation $j: \mathfrak{z} \rightarrow s o(\mathfrak{v})$ by $j(Z) X=(\operatorname{ad} X)^{*} Z$ for $Z \in \mathfrak{z}$ and $X \in \mathfrak{v}$. Equivalently, for each $Z \in \mathfrak{z}, j(Z): \mathfrak{v} \rightarrow \mathfrak{v}$ is the skew-symmetric linear transformation defined by

$$
\langle j(Z) X, Y\rangle=\langle Z,[X, Y]\rangle
$$

for all $X, Y$ in $\mathfrak{v}$. Here $\operatorname{ad} X(Y)=[X, Y]$ for all $X, Y \in \mathfrak{g}$, and $(\operatorname{ad} X)^{*}$ denotes the (metric) adjoint of $\operatorname{ad} X$.

By skew-symmetry, $j(Z)$ has $\operatorname{dim}_{\mathbb{R}}(\mathfrak{v})$ purely complex eigenvalues counting (algebraic) multiplicities and the nonzero eigenvalues occur in complex conjugate pairs; the eigenvalues of $j(Z)^{2}$ are then real and nonpositive. If $j(Z)$ is nonsingular for some $Z$ in $\mathfrak{z}$, then the (real) dimension of $\mathfrak{v}$ is even.

Each two-step nilpotent metric Lie algebra carries with it the $j$ operator. On the other hand, given inner product spaces $\mathfrak{v}$ and $\mathfrak{z}$ and a linear transformation $j: \mathfrak{z} \rightarrow s o(\mathfrak{v})$, one can define a two-step nilpotent metric Lie algebra $(\mathfrak{v} \oplus \mathfrak{z},\langle\rangle$,$) by$ requiring that $\mathfrak{z}$ be central, that $\oplus$ be an orthogonal direct sum, and by defining the Lie bracket [ , ] via (2.3). All two-step nilpotent metric Lie algebras are determined this way.

2.4 Definitions. Let $(\mathfrak{g},\langle\rangle$,$) be a two-step nilpotent metric Lie algebra and let$ $Z \in \mathfrak{z}$.

(1) Let $\mu(Z)$ denote the number of distinct eigenvalues of $j(Z)^{2}$. For ease of notation, we write $\mu$ rather than $\mu(Z)$ when $Z$ is understood.

(2) Let $-\vartheta_{1}(Z)^{2},-\vartheta_{2}(Z)^{2}, \ldots,-\vartheta_{\mu}(Z)^{2}$ denote the $\mu$ distinct eigenvalues of $j(Z)^{2}$, with the assumption that $0 \leq \vartheta_{1}(Z)<\vartheta_{2}(Z)<\cdots<\vartheta_{\mu}(Z)$. The distinct eigenvalues of $j(Z)$ are then $\left\{ \pm \vartheta_{1}(Z) i, \ldots, \pm \vartheta_{\mu}(Z) i\right\}$.

2.5 Notation. Let $V+Z \in \mathfrak{g}$, with $V \in \mathfrak{v}, Z \in \mathfrak{z}$. Denote by $Z_{V}$ the orthogonal projection of $Z$ onto the subalgebra $[V, \mathfrak{g}]=\{[V, X]: X \in \mathfrak{g}\} \subset \mathfrak{z}$. Denote by $Z_{V}^{\perp}=Z-Z_{V}$, so that $Z_{V}^{\perp}$ is the component of $Z$ that is orthogonal to $[V, \mathfrak{g}]$.

We are now ready to state the main theorems of this paper.

2.6 Main Theorem. Let $(\Gamma \backslash G, g)$ be a Riemannian two-step nilmanifold. With notation as above, $(\Gamma \backslash G, g)$ satisfies the Clean Intersection Hypothesis if and only if for all $V+Z \in \log \Gamma$ and for all $m=1, \ldots, \mu\left(Z_{V}^{\perp}\right)$,

$$
\vartheta_{m}\left(Z_{V}^{\perp}\right) \notin \mathbb{Q}^{+} \pi \text {. }
$$

Theorem 2.6 actually follows directly from the more precise statement Theorem 3.1 below. The proof of Theorem 3.1 together with further background information of two-step nilmanifolds is the focus of Section 3.

2.7 Example: Heisenberg manifold (continued). We continue with example 1.20, where $H$ is the three-dimensional Heisenberg Lie group and $g$ is the unique left-invariant metric on $H$ such that $\{X, Y, Z\}$ is an orthonormal basis. For this metric,

$$
j(c Z)=\left(\begin{array}{cc}
0 & -c \\
c & 0
\end{array}\right)
$$

and $\vartheta_{1}(c Z)=|c|$ for all $c \in \mathbb{R}$. Let $\Gamma$ be any cocompact, discrete subgroup of $H$ such that $2 \pi k Z \in \log \Gamma \cap \mathfrak{z}$ for some $k \in \mathbb{Z}^{+}$, such as the one defined in Example 
1.20. Then $\vartheta_{1}(2 \pi k Z)=2 \pi k$, and by Theorem $2.6,(\Gamma \backslash G, g)$ does not satisfy the Clean Intersection Hypothesis.

2.8 Corollary. Let $G$ be a simply-connected, two-step nilpotent Lie group with cocompact, discrete subgroup $\Gamma$. There exist left invariant metrics on $G$ such that $(\Gamma \backslash G, g)$ fails to satisfy the Clean Intersection Hypothesis.

Proof of Corollary 2.8. Let $G$ be a simply-connected two-step nilpotent Lie group. Let $\Gamma$ be a cocompact, discrete subgroup of $\Gamma$. Let $Z^{*} \in \log \Gamma \cap \mathfrak{z}$. Let $g$ be any left invariant metric on $G$ such that $Z^{*}$ is a unit vector and such that $j\left(Z^{*}\right) \not \equiv 0$. Then $j\left(Z^{*}\right)$ has eigenvalues $\left\{ \pm \vartheta_{1}\left(Z^{*}\right), \ldots, \pm \vartheta_{\mu}\left(Z^{*}\right)\right\}$. Pick one nonzero eigenvalue of $j\left(Z^{*}\right)$, say $\vartheta_{m}\left(Z^{*}\right)$. Let $\lambda$ be such that $\lambda \vartheta_{m}\left(Z^{*}\right) \in \mathbb{Q} \pi$. Let $g_{\lambda}$ be any left invariant metric on $G$ such that

$$
\frac{1}{\sqrt{\lambda}} Z^{*}
$$

is a unit vector and the metric on the orthogonal complement of the center remains unchanged. Let $j_{\lambda}$ denote the $J$-operator in the metric $g_{\lambda}$ with eigenvalues denoted $\vartheta_{m}^{\lambda}$. One easily calculates that $j_{\lambda}\left(Z^{*}\right)=\lambda j\left(Z^{*}\right)$, and $\vartheta_{m}^{\lambda}\left(Z^{*}\right)=\lambda \vartheta_{m}\left(Z^{*}\right) \in \mathbb{Q} \pi$. By Theorem 2.6, $\left(\Gamma \backslash G, g_{\lambda}\right)$ does not satisfy the Clean Intersection Hypothesis.

2.9 Corollary. If $(\Gamma \backslash G, g)$ is a two-step Riemannian nilmanifold that satisfies the Clean Intersection Hypothesis, then there exists a sequence of metrics $g_{k}$ on $\Gamma \backslash G$ arising from a left invariant metric on $G, \lim _{k \rightarrow \infty} g_{k}=g$, such that $\left(\Gamma \backslash G, g_{k}\right)$ fails to satisfy the Clean Intersection Hypothesis.

Proof of Corollary 2.9. This follows from the process described in the previous corollary, by choosing a sequence $\lambda_{k}$ such that $\lambda_{k} \vartheta_{m}\left(Z^{*}\right) \in \mathbb{Q} \pi$ and $\lim _{k \rightarrow \infty} \lambda_{k}=$ 1.

In other words, "unclean" two-step nilmanifolds are dense among two-step nilmanifolds. However, the family of two-step nilmanifolds that satisfy the hypotheses of Theorem 2.6 are clearly measure zero in the family of all two-step nilmanifolds.

2.10 Theorem. Generic two-step nilmanifolds satisfy the Clean Intersection Hypothesis.

2.11. See [G1] for the first examples and Gt2] for further examples of pairs of two-step nilmanifolds that are isospectral on functions but which have different multiplicities in the length spectrum. It is interesting to note that the lengths that produce different multiplicities in the isospectral pairs are not those associated to the "unclean" lengths, described above. Thus, the multiplicity phenomena does not appear related to the Clean Intersection Hypothesis. See Gt6] for results comparing the wave invariants of these examples.

2.12 Remark. In special cases, using Riemannian submersions, one should be able to construct higher-step nilmanifolds that fail the Clean Intersection Hypothesis. Technical difficulties arise in proving that the clean intersection hypothesis must respect Riemannian submersions.

\section{Proof of the MaIn THEOREM}

Recall that throughout this paper, a prime (e.g. $\left.X^{\prime}(t)\right)$ denotes derivation with respect to the $t$ or $r$ variable, and a dot (e.g. $\dot{\alpha}(s))$ denotes derivation with respect to the $s$ variable. Also recall that elements with tildes such as $\tilde{p}, v_{\tilde{p}}$, denote elements 
in $G$ and $\tilde{\mathfrak{X}}$, respectively; elements without tildes such as $p$ and $v_{p}$ denote the image of $\tilde{p}$ and $v_{\tilde{p}}$ in $\Gamma \backslash G$ and $\mathfrak{X}$, respectively, under the canonical mapping $\pi: \tilde{\mathfrak{X}} \rightarrow \mathfrak{X}$. The element $e$ is the identity element of $G$.

The purpose of this section is to prove the following theorem, from which Theorem 2.6 follows directly.

3.1 Theorem. Let $(\Gamma \backslash G, g)$ be a Riemannian two-step nilmanifold. With notation as established in Section 1 and Section $2,(\Gamma \backslash G, g)$ satisfies the Clean Intersection Hypothesis if and only if for all $\gamma=\exp (V+Z) \in \Gamma$ and for all $m=1, \ldots, \mu\left(Z_{V}^{\perp}\right)$, $\vartheta_{m}\left(Z_{V}^{\perp}\right) \notin 2 \pi \mathbb{Z}^{+}$. In particular, if $\tau \in \operatorname{spec}_{L}(\Gamma \backslash G, g)$ and there exists $\gamma \in \Gamma$ and $v_{p} \in F\left(\tau, c_{\gamma}\right)$ such that $T_{v_{p}} F\left(\tau, c_{\gamma}\right) \neq F P S\left(\tau, v_{p}\right)$, then $\tau=\left|V+Z_{V}^{\perp}\right|$ and there exists $m \in\left\{1, \ldots, \mu\left(Z_{V}^{\perp}\right)\right\}$ such that $\vartheta_{m}\left(Z_{V}^{\perp}\right) \in 2 \pi \mathbb{Z}^{+}$. On the other hand, if $\gamma=$ $\exp (V+Z) \in \Gamma$ and $\vartheta_{m}\left(Z_{V}^{\perp}\right) \in 2 \pi \mathbb{Z}^{+}$for some $m=1, \ldots, \mu\left(Z_{V}^{\perp}\right)$, letting $\tau=$ $\left|V+Z_{V}^{\perp}\right|$, then $F\left(\tau, c_{\gamma}\right) \neq \emptyset$ and $T_{v_{p}} F\left(\tau, c_{\gamma}\right) \neq F P S\left(\tau, v_{p}\right)$.

To see that Theorem 3.1 implies Theorem 2.6, let $(\Gamma \backslash G, g)$ be a Riemannian two-step nilmanifold. Let $\gamma=\exp (V+Z) \in \Gamma$ such that $\vartheta_{m}\left(Z_{V}^{\perp}\right) \in 2 \pi \mathbb{Q}^{+}$. Let $h, h^{\prime} \in \mathbb{Z}^{+}$such that $\vartheta_{m}\left(Z_{V}^{\perp}\right)=\frac{h}{h^{\prime}} \pi$. Then $2 h^{\prime}(V+Z)=\log \left(\gamma^{2 h^{\prime}}\right)$ is also in $\log \Gamma$. Note that $\left(2 h^{\prime}(V+Z)\right)_{V}^{\perp}=2 h^{\prime} Z_{V}^{\perp}$, and $\vartheta_{m}\left(2 h^{\prime} Z_{V}^{\perp}\right)=2 h^{\prime} \vartheta_{m}\left(Z_{V}^{\perp}\right)=2 h \pi \in 2 \pi \mathbb{Z}^{+}$. So, there exists $\gamma=\exp (V+Z) \in \Gamma$ such that $\vartheta_{m}\left(Z_{V}^{\perp}\right) \in 2 \pi \mathbb{Q}^{+}$if and only if there exists $\gamma^{\prime}=\exp \left(V^{\prime}+Z^{\prime}\right) \in \Gamma$ such that $\vartheta_{m}\left(Z_{V}^{\prime}\right) \in 2 \pi \mathbb{Z}^{+}$.

We need additional information and notation about two-step nilmanifolds before proceeding with the proof of Theorem 3.1.

We continue the notation of Section 1 and Section 2.

3.2 Notation. (1) Let $W_{m}(Z)$ denote the invariant subspace of $j(Z)$ corresponding to $\vartheta_{m}(Z), m=1, \ldots, \mu$. Then $j(Z)_{\mid W_{m}(Z)}^{2}=-\vartheta_{m}(Z)^{2} \operatorname{Id}_{\mid W_{m}(Z)}$; i.e., $W_{m}(Z)$ is the eigenspace of $j(Z)^{2}$ with eigenvalue $-\vartheta_{m}(Z)^{2}$. In particular, if $\vartheta_{1}(Z)=0$, then $W_{1}(Z)=\operatorname{ker} j(Z)$. By the skew-symmetry of $j(Z)$, $\mathfrak{v}$ is the orthogonal direct sum of the invariant subspaces $W_{m}(Z)$ and we write

$$
\mathfrak{v}=\bigoplus_{m=1}^{\mu} W_{m}(Z)
$$

(2) Note that if $\vartheta_{m}(Z) \neq 0$, then

$$
j(Z)_{\mid W_{m}(Z)}^{-1}=\frac{-1}{\vartheta_{m}(Z)^{2}} j(Z)_{\mid W_{m}(Z)}
$$

and for $m=1, \ldots, \mu$,

$$
e^{s J}=\cos \left(s \vartheta_{m}\right) \operatorname{Id}+\frac{\sin \left(s \vartheta_{m}\right)}{\vartheta_{m}} J \quad \text { on } W_{m}(Z),
$$

where $J=j(Z)$ and $\vartheta_{m}=\vartheta_{m}(Z)$.

3.3 Notation. Let $X_{0}+Z_{0}$ be a vector in $\mathfrak{n}$ with $X_{0} \in \mathfrak{v}$ and $Z_{0} \in \mathfrak{z}$.

(1) Define $X_{1}$ and $X_{2}$ by $X_{0}=X_{1}+X_{2}$ such that $X_{1} \in \operatorname{ker} j\left(Z_{0}\right)$ and $X_{2} \perp$ $\operatorname{ker} j\left(Z_{0}\right)$.

(2) Let $\xi_{m}$ denote the component of $X_{2}$ in $W_{m}\left(Z_{0}\right)$, for each $m$. We write $X_{2}=\sum_{m} \xi_{m}$. Note that if $W_{1}\left(Z_{0}\right)=\operatorname{ker} j\left(Z_{0}\right)$, then $\xi_{1}=0$. When necessary, we assume $j(Z)^{-1} \xi_{1}=0$ if $\vartheta_{1}(Z)=0$. 
3.4 Definitions. (1) For $V$ in $\mathfrak{v}$, define $P_{V}: \mathfrak{z} \rightarrow[V, \mathfrak{g}]$ as orthogonal projection onto $[V, \mathfrak{g}]$. Define $P_{V}^{\perp}: \mathfrak{z} \rightarrow[V, \mathfrak{g}]^{\perp}$ as projection onto $[V, \mathfrak{g}]^{\perp}$, the orthogonal complement of $[V, \mathfrak{g}]$ in $\mathfrak{z}$. For $V \in \mathfrak{v}$ and $Z \in \mathfrak{z}$, define $Z_{V}=P_{V}(Z)$ and $Z_{V}^{\perp}=P_{V}^{\perp}(Z)$, which is consistent with the notation of 2.5.

(2) With notation as above, define $K: \mathfrak{g} \rightarrow \mathfrak{z}$ by

$$
K\left(X_{0}+Z_{0}\right)=Z_{0}+\frac{1}{2} \sum_{m}\left[j\left(Z_{0}\right)^{-1} \xi_{m}, \xi_{m}\right] .
$$

(3) Set $K_{V}=P_{V} \circ K$ and $K_{V}^{\perp}=P_{V}^{\perp} \circ K$.

Note that

$$
\left\langle Z_{0}, K\left(X_{2}+Z_{0}\right)\right\rangle=\left|Z_{0}\right|^{2}+\frac{1}{2} \sum_{m}\left\langle Z_{0},\left[j\left(Z_{0}\right)^{-1} \xi_{m}, \xi_{m}\right]\right\rangle=\left|Z_{0}\right|^{2}+\frac{1}{2}\left|X_{2}\right|^{2} .
$$

Thus $K\left(X_{2}+Z_{0}\right)=0$ if and only if $X_{2}+Z_{0}=0$, and, $K\left(X_{2}+Z_{0}\right)=Z_{0}$ if and only if $X_{2}=0$. Note also that if $Z_{0} \perp[V, \mathfrak{g}], Z_{0} \neq 0$, then $K_{V}^{\perp}\left(X_{2}+Z_{0}\right) \neq 0$.

3.5 Geodesic flow of two-step nilmanifolds. By left invariance,

$$
\tilde{\Phi}\left(t, v_{\tilde{p}}\right)=\tilde{p} \cdot \tilde{\Phi}\left(t, v_{e}\right)
$$

for all $\tilde{p} \in G$. So it is enough to calculate the geodesic flow of $(G, g)$ at the identity $e \in G$. Let $v_{e} \in T_{e} G \cong \mathfrak{g}$, with $v_{e}=X_{1}+X_{2}+Z_{0}$ as in Notation 3.3. By [E1, Prop $3.5]$

$$
\sigma_{v_{e}}(t)=\tilde{\Phi}\left(t,\left(e, X_{1}+X_{2}+Z_{0}\right)\right)=\left(\exp (X(t)+Z(t)), X^{\prime}(t)+Z_{0}\right),
$$

where $X_{2}=\sum_{m} \xi_{m}$ with $\xi_{m} \in W_{m}\left(Z_{0}\right), J=j\left(Z_{0}\right), \vartheta_{m}=\vartheta_{m}\left(Z_{0}\right)$, and

$$
\begin{aligned}
X(t) & =t X_{1}+\left(e^{t J}-\mathrm{Id}\right)\left(J^{-1} X_{2}\right) \\
& =t X_{1}+\sum_{m} \frac{1-\cos \left(t \vartheta_{m}\right)}{\vartheta_{m}}\left(\frac{1}{\vartheta_{m}} J \xi_{m}\right)+\sum_{m} \frac{\sin \left(t \vartheta_{m}\right)}{\vartheta_{m}} \xi_{m}, \\
X^{\prime}(t) & =X_{1}+e^{t J} X_{2} \\
& =X_{1}+\sum_{m} \sin \left(t \vartheta_{m}\right)\left(\frac{1}{\vartheta_{m}} J \xi_{m}\right)+\sum_{m} \cos \left(t \vartheta_{m}\right) \xi_{m}, \\
Z(t) & =\int_{0}^{t}\left(Z_{0}+\frac{1}{2}\left[X(r), X^{\prime}(r)\right]\right) d r .
\end{aligned}
$$

For a more detailed expression of $Z(t)$, involving $X_{1}, \xi_{m}$, and $\vartheta_{m}$, see [E1, Prop 3.5].

3.6 Theorem ([E1]). Let $G$ be a simply-connected two-step nilpotent Lie group with left invariant metric $g$ and metric Lie algebra $(\mathfrak{g},\langle\rangle$,$) . Let X_{0}+Z_{0}$ be a unit vector in $\mathfrak{g}$ with $X_{0} \in \mathfrak{v}, Z_{0} \in \mathfrak{z}$. We write $X_{0}=X_{1}+X_{2}, X_{2}=\sum_{m} \xi_{m}$ as in (3.3). Given a unit speed geodesic $\sigma$ with $\sigma(0)=e$ and $\dot{\sigma}(0)=X_{0}+Z_{0}$, the following statements hold:

(1) Assume there exists $\omega>0$ and $\phi \in G$ such that $\phi \sigma(s)=\sigma(s+\omega)$ for all s. Let $\phi=\exp (V+Z)$, where $V \in \mathfrak{v}$ and $Z \in \mathfrak{z}$. Then $X(\omega)=V=\omega X_{1}$ and $\xi_{m} \neq 0$ implies $\omega \vartheta_{m}\left(Z_{0}\right) \in 2 \pi \mathbb{Z}^{+}$for all $m=1, \ldots, \mu$. Furthermore, $Z(\omega)=Z=\omega K\left(X_{2}+Z_{0}\right)+\left[V, j\left(Z_{0}\right)^{-1} X_{2}\right]$.

(2) The value $\omega>0$ satisfies $\sigma(\omega) \sigma(s)=\sigma(s+\omega)$ for all $s$ if and only if $Z_{0} \perp[\log \sigma(\omega), \mathfrak{g}]$. 
3.7 Theorem ([GtM1]). Let $G$ be a simply-connected two-step nilpotent Lie group with left invariant metric $g$. Let $(\mathfrak{g},\langle\rangle$,$) be the associated metric Lie algebra. With$ notation as above, let $\gamma=\exp \left(V+Z_{V}+Z_{V}^{\perp}\right)$ be an element of $G$. The periods of $\gamma$ are precisely

$$
\left\{\sqrt{|V|^{2}+\frac{\left|Z_{V}^{\perp}\right|^{2}}{\left|K_{V}^{\perp}\left(X_{2}+Z_{0}\right)\right|^{2}}}: X_{2}+Z_{0} \text { satisfy (i)-(iv) below }\right\},
$$

where $\frac{\left|Z_{V}^{\perp}\right|}{\left|K_{\bar{V}}^{\perp}\left(X_{2}+Z_{0}\right)\right|}=0$ if $Z_{V}^{\perp}=0$. Given $X_{2} \in \mathfrak{v}$ and $Z_{0} \in \mathfrak{z}$, the conditions referred to in $(\dagger)$ are the following:

(i) $\left|X_{2}+Z_{0}\right|=1$ or $X_{2}+Z_{0}=0$,

(ii) $V \in \operatorname{ker} j\left(Z_{0}\right)$ and $X_{2} \perp \operatorname{ker} j\left(Z_{0}\right)$,

(iii) $Z_{V}^{\perp} \in \operatorname{span}_{\mathbb{R}^{+}}\left\{K_{V}^{\perp}\left(X_{2}+Z_{0}\right)\right\}$,

(iv) for all $m$ such that $\xi_{m} \neq 0, \frac{\left|Z_{V}^{\perp}\right| \vartheta_{m}\left(Z_{0}\right)}{2 \pi\left|K_{V}^{\perp}\left(X_{2}+Z_{0}\right)\right|} \in \mathbb{Z}$.

We need information about the initial velocities that achieve the periods in Theorem 3.7. We now show:

3.8 Theorem. Let $G$ be a simply-connected two-step nilpotent Lie group with left invariant metric g. Let $\gamma=\exp (V+Z) \in G$ and let $\tau>0$. With notation as in Section 1 and Section 2,

$$
\begin{array}{r}
\tilde{F}(\tau, \gamma)=\left\{v_{\tilde{p}}: v=\frac{1}{\tau}\left(V+\sqrt{\tau^{2}-|V|^{2}}\left(X_{2}+Z_{0}\right)\right)\right. \\
\left.\tilde{p} \text { and } X_{2}+Z_{0} \text { satisfy (i)-(v) below }\right\} .
\end{array}
$$

Given $\tilde{p} \in G, X_{2} \in \mathfrak{v}$ and $Z_{0} \in \mathfrak{z}$, the conditions referred to in (W) are the following:

(i) $\left|X_{2}+Z_{0}\right|=1$ or $X_{2}+Z_{0}=0$,

(ii) $V \in \operatorname{ker} j\left(Z_{0}\right)$ and $X_{2} \perp \operatorname{ker} j\left(Z_{0}\right)$,

(iii) $K_{V}^{\perp}\left(X_{2}+Z_{0}\right)=\frac{1}{\sqrt{\tau^{2}-|V|^{2}}} Z_{V}^{\perp}$, where $\frac{1}{\sqrt{\tau^{2}-|V|^{2}}} Z_{V}^{\perp}=0$ if $Z_{V}^{\perp}=0$,

(iv) for all $m$ such that $\xi_{m} \neq 0, \frac{\sqrt{\tau^{2}-|V|^{2}}}{2 \pi} \vartheta_{m}\left(Z_{0}\right) \in \mathbb{Z}$, and

(v) $[V, \log \tilde{p}]+Z_{V}=\left[V, j\left(Z_{0}\right)^{-1} X_{2}\right]+\sqrt{\tau^{2}-|V|^{2}} K_{V}\left(X_{2}+Z_{0}\right)$.

Note that $\tilde{F}(\tau, \gamma)=\emptyset$ if no such $\tilde{p}, X_{2}, Z_{0}$ exist.

\subsection{Remarks. $\quad$ a. Note that $X_{2}+Z_{0}$ satisfies (i)-(iv) of Theorem 3.7, and thus} produces the period

$$
\tau=\sqrt{|V|^{2}+\frac{\left|Z_{V}^{\perp}\right|^{2}}{\left|K_{V}^{\perp}\left(X_{2}+Z_{0}\right)\right|^{2}}},
$$

if and only if $X_{2}+Z_{0}$ satisfies (i)-(iv) of Theorem 3.8 for this value of $\tau$. The additional condition (v) in Theorem 3.8 corresponds to the property a base point of a geodesic $\sigma_{v_{\tilde{p}}}$ that achieves the period $\tau$ must satisfy. Note that if $\gamma \in Z(G)$, then $V=0$ and condition (v) of Theorem 3.8 is satisfied for any point $\tilde{p} \in G$. 
b. By [E1, Prop. 4.5(2)], $\tau=\left|V+Z_{V}^{\perp}\right|$ is always a period of $\gamma$, and for this value of $\tau, v=\frac{1}{\tau}\left(V+Z_{V}^{\perp}\right)$, i.e., $X_{2}=0, Z_{0}=\frac{1}{\left|Z_{V}^{\perp}\right|} Z_{V}^{\perp}$, and $K\left(X_{2}+Z_{0}\right)=\frac{1}{\left|Z_{V}^{\perp}\right|} Z_{V}^{\perp}$. This also follows from Theorem 3.7. In this case, condition (v) becomes $[V, \log \tilde{p}]+Z_{V}=0$. One may compute that

$$
\left\{\tilde{p} \in G:[\log \tilde{p}, V]=Z_{V}\right\}=C(\gamma, G) \tilde{q}=\tilde{q} C(\gamma, G),
$$

where $\tilde{q}$ is any fixed element in $G$ such that

$$
\tilde{q}^{-1} \gamma \tilde{q}=\exp \left(V+Z_{V}^{\perp}\right) .
$$

To see this, note that by $(2.1),[V, \log \tilde{p}]=[V, \log \tilde{q}]$ if and only if $\tilde{p} \tilde{q}^{-1} \in$ $C(\gamma, G)$. Thus for $\tau=\left|V+Z_{V}^{\perp}\right|$,

$$
\tilde{F}(\tau, \gamma)=C(\gamma, G) \tilde{q} \times \frac{1}{\tau}\left(V+Z_{V}^{\perp}\right) .
$$

Proof of Theorem 3.8. Recall that $K\left(\lambda\left(X_{2}+Z_{0}\right)\right)=\lambda K\left(X_{2}+Z_{0}\right)$ for all $\lambda \in \mathbb{R}$ and $\theta_{m}\left(\lambda Z_{0}\right)=|\lambda| \theta_{m}\left(Z_{0}\right)$ for all $\lambda \neq 0$ and for all $m=1, \ldots, \mu\left(Z_{0}\right)$. Also, $j(c Z)^{-1}=$ $\frac{1}{c} j(Z)^{-1}$ for all $c \neq 0$.

Let $\sigma(s)$ be a unit speed geodesic on $(G, g)$ such that $\gamma \sigma(s)=\sigma(s+\tau)$ for all $s$. Let $\dot{\sigma}(0)=v_{\tilde{p}}$ and $\tilde{p}=\sigma(0)$. Then $\alpha(s)=\tilde{p}^{-1} \sigma(s)$ is a unit speed geodesic such that $\tilde{p}^{-1} \gamma \tilde{p} \alpha(s)=\alpha(s+\tau)$ for all $s$; i.e., $\alpha(s)$ is a unit speed geodesic with period $\tau$. Note that by $(2.1)$,

$$
\log \left(\tilde{p}^{-1} \gamma \tilde{p}\right)=V+Z_{V}+Z_{V}^{\perp}+[V, \log \tilde{p}] .
$$

Let $\alpha(s)=\exp (X(s)+Z(s))$ as in Subsection 3.5. Denote the initial velocity of $\alpha(s)$ by $v=X_{1}^{\prime}+X_{2}^{\prime}+Z_{0}^{\prime}$, where $Z_{0}^{\prime} \in \mathfrak{z}, X_{1}^{\prime} \in \operatorname{ker} j\left(Z_{0}^{\prime}\right)$ and $X_{2}^{\prime} \perp \operatorname{ker} j\left(Z_{0}^{\prime}\right)$. By part (1) of Theorem 3.6,

$$
\tau X_{1}^{\prime}=V
$$

and

$$
\log \left(\tilde{p}^{-1} \gamma \tilde{p}\right)=V+\tau K\left(X_{2}^{\prime}+Z_{0}^{\prime}\right)+\left[V, j\left(Z_{0}^{\prime}\right)^{-1} X_{2}^{\prime}\right] .
$$

Thus

$$
Z_{V}+[V, \log \tilde{p}]=\tau K_{V}\left(X_{2}^{\prime}+Z_{0}^{\prime}\right)+\left[V, j\left(Z_{0}^{\prime}\right)^{-1} X_{2}^{\prime}\right]
$$

and

$$
Z_{V}^{\perp}=\tau K_{V}^{\perp}\left(X_{2}^{\prime}+Z_{0}^{\prime}\right)
$$

Note that if $Z_{V}^{\perp}=0$, then $K_{V}^{\perp}\left(X_{2}^{\prime}+Z_{0}^{\prime}\right)=0$, which by the remarks following Definitions 3.4 implies $Z_{0}^{\prime}=0, X_{2}^{\prime}=0$ and $\left[V, j\left(Z_{0}^{\prime}\right)^{-1} X_{2}^{\prime}\right]=0$. In this case, $\tau=|V|$ and $v=\frac{V}{|V|}$, as desired. Note that conditions (i)-(iv) are automatically satisfied. Also, condition (v) follows from the fact that $\sqrt{\tau^{2}-|V|^{2}}=0$.

So we may assume $Z_{V}^{\perp} \neq 0$, which implies $X_{2}^{\prime}+Z_{0}^{\prime} \neq 0$.

If $V \neq 0$, then $\tau=|V| /\left|X_{1}^{\prime}\right|$ and since $v$ is a unit vector,

$$
\left|X_{2}^{\prime}+Z_{0}^{\prime}\right|=\frac{\tau}{\sqrt{\tau^{2}-|V|^{2}}} .
$$

Note that this holds even if $V=0$, since then $X_{1}^{\prime}=0$ and $X_{2}^{\prime}+Z_{0}^{\prime}$ is a unit vector.

Let $X_{2}+Z_{0}=\frac{X_{2}^{\prime}+Z_{0}^{\prime}}{\left|X_{2}^{\prime}+Z_{0}^{\prime}\right|}$, so that conditions (i) and (ii) of ( $V=\tau X_{1}^{\prime}$.

Then

$$
X_{2}^{\prime}+Z_{0}^{\prime}=\frac{\sqrt{\tau^{2}-|V|^{2}}}{\tau}\left(X_{2}+Z_{0}\right)
$$


which implies

$Z_{V}^{\perp}=\tau K_{V}^{\perp}\left(X_{2}^{\prime}+Z_{0}^{\prime}\right)=\tau K_{V}^{\perp}\left(\left(\frac{\sqrt{\tau^{2}-|V|^{2}}}{\tau}\right)\left(X_{2}+Z_{0}\right)\right)=\sqrt{\tau^{2}-|V|^{2}} K_{V}^{\perp}\left(X_{2}+Z_{0}\right)$,

and condition (iii) of ( is satisfied.

By part (1) of Theorem 3.6, we must have $\tau \vartheta_{m}\left(Z_{0}^{\prime}\right) \in 2 \pi \mathbb{Z}$ for all $m$ such that $\xi_{m} \neq 0$. This implies

$$
\tau \vartheta_{m}\left(Z_{0}^{\prime}\right)=\sqrt{\tau^{2}-|V|^{2}} \vartheta_{m}\left(Z_{0}\right) \in 2 \pi \mathbb{Z}
$$

for all $m$ such that $\xi_{m} \neq 0$, and condition (iv) of (

Finally,

$$
Z_{V}+[V, \log \tilde{p}]=\tau K_{V}\left(X_{2}^{\prime}+Z_{0}^{\prime}\right)+\left[V, j\left(Z_{0}^{\prime}\right)^{-1} X_{2}^{\prime}\right]
$$

implies condition (v) of ( $)$ after normalizing to $X_{2}+Z_{0}$.

Thus if $v_{\tilde{p}} \in \tilde{F}(\tau, \gamma)$, then $v_{\tilde{p}}$ mast satisfy the conditions of ( $\left.\mathbf{\Psi}\right)$.

To prove equality, let $v_{\tilde{p}}$ satisfy the conditions of $(\mathbf{W})$. Let $\alpha(s)$ be the unit speed geodesic with initial velocity $v_{\tilde{p}}$. We must show $\gamma \alpha(s)=\alpha(s+\tau)$ for all $s$. Recall that $\gamma=\exp (V+Z)$, where $V \in \mathfrak{v}$ and $Z \in \mathfrak{z}$.

Left translate $\alpha(s)$ to $e$, by defining $\sigma(s):=\tilde{p}^{-1} \alpha(s)$ for all $s$. This new curve $\sigma(s)$ is a geodesic since left translations are isometries. We must now show $\tilde{p}^{-1} \gamma \tilde{p} \sigma(s)=$ $\sigma(s+\tau)$ for all $s$.

To do this, we must first show $\sigma(\tau)=\tilde{p}^{-1} \gamma \tilde{p}$. Let $\sigma(s)=\exp (X(s)+Z(s))$ as in Subsection 3.5. By (2.1), we must show that $X(\tau)=V$ and $Z(\tau)=Z+[V, \log \tilde{p}]$. By the conditions of $(\mathbf{W}), v=X_{1}^{\prime}+X_{2}^{\prime}+Z_{0}^{\prime}$ where $Z_{0}^{\prime}=\frac{\sqrt{\tau^{2}-|V|^{2}}}{\tau} Z_{0}, X_{2}^{\prime}=\frac{\sqrt{\tau^{2}-|V|^{2}}}{\tau} X_{2}$, $X_{1}^{\prime}=\frac{1}{\tau} V$, and $X_{2}+Z_{0}$ satisfies the conditions of ( By the geodesic equations in Subsection 3.5 and condition (iv) of $(\mathbf{W}), X(\tau)=\tau X_{1}^{\prime}=V$. Note that by condition (ii) of ( $\mathbf{\Psi})$, this implies $[\log \sigma(\tau), \mathfrak{g}] \perp Z_{0}^{\prime}$, so by part (2) of Theorem 3.6, $\sigma(s)$ is periodic by $\sigma(\tau)=\tilde{p}^{-1} \gamma \tilde{p}$.

We may now use condition (1) of Theorem 3.6, so that $Z(\tau)=\tau K\left(X_{2}^{\prime}+Z_{0}^{\prime}\right)+$ $\left[V, j\left(Z_{0}^{\prime}\right)^{-1} X_{2}^{\prime}\right]$. After normalizing to $X_{2}+Z_{0}$ as above, we obtain

$$
Z(\tau)=\left(\sqrt{\tau^{2}-|V|^{2}} K_{V}\left(X_{2}+Z_{0}\right)+\left[V, j\left(Z_{0}\right)^{-1} X_{2}\right]\right)+\sqrt{\tau^{2}-|V|^{2}} K_{V}^{\perp}\left(X_{2}+Z_{0}\right) .
$$

Using conditions (iii) and (v) of ( $\mathbf{\Psi})$, we see that $Z(\tau)=[V, \log \tilde{p}]+Z$, as desired.

3.10 Notation: Fixed points on two-step nilmanifolds. With notation as above, let $\gamma=\exp (V+Z) \in \Gamma$, with $Z=Z_{V}+Z_{V}^{\perp}$. By (2.1) there exists $\tilde{q} \in G$ such that

$$
\tilde{q}^{-1} \gamma \tilde{q}=\exp \left(V+Z_{V}^{\perp}\right)
$$

We consider $\tilde{q}$ and $\gamma$ (hence, $V, Z, Z_{V}, Z_{V}^{\perp}$ ) fixed in what follows.

Let $C(\gamma, G)$ denote the centralizer of $\gamma$ in $G$. One easily calculates from (2.1) that

$$
C(\gamma, G)=\{\exp (X): X \in \mathfrak{g},[X, V]=0\}
$$

Let

$$
\tilde{p}=x \tilde{q} a,
$$

where $x \in C(\gamma, G)$ and $a$ is an arbitrary element in $G$.

We identify

$$
v_{\tilde{p}}=(\tilde{p}, v)
$$


as in (1.9) and let

$$
\alpha(s)=\left(\tilde{p} \exp \left(a_{1}(s)\right), a_{2}(s)\right) \in \tilde{\mathfrak{X}} \cong G \times S^{n-1}
$$

with

so that

$$
a_{1}(0)=0 \quad \text { and } \quad a_{2}(0)=v \text {, }
$$

$$
\alpha(0)=(\tilde{p}, v)=v_{\tilde{p}}
$$

Since $\exp _{*_{e}}=\mathrm{Id}$, we have $\frac{d}{d s \mid 0} \tilde{p} \cdot \exp \left(a_{1}(s)\right)=L_{\tilde{p} *}\left(\dot{a}_{1}(0)\right)$, and

$$
\dot{\alpha}(0)=\left(\dot{a}_{1}(0), \dot{a}_{2}(0)\right)_{v_{\tilde{p}}} \in T_{v_{\tilde{p}}} \tilde{\mathfrak{X}} \cong T_{\tilde{p}} G \times T_{v} S^{n-1} .
$$

For $v_{p} \in F(\tau, c)$, we seek conditions so that $\pi_{*} \dot{\alpha}(0)$ is a fixed point of $\Phi_{\tau *_{v_{p}}}$. Equivalently by Proposition 1.18 , for $v_{\tilde{p}} \in \tilde{F}(\tau, \gamma)$ we calculate conditions so that

$$
\tilde{\Phi}_{\tau *_{\tilde{p}}}(\dot{\alpha}(0))=L_{\gamma *}(\dot{\alpha}(0)) \text {. }
$$

Note that this implies $\tilde{\Phi}_{\tau}(\alpha(0))=\gamma \cdot \alpha(0)$.

For each $s$, we orthogonally decompose

$$
a_{2}(s)=X_{0}^{s}+Z_{0}^{s},
$$

where $Z_{0}^{s}$ is the central component of $a_{2}(s)$ and $X_{0}^{s} \perp Z_{0}^{s}$, so that

$$
v=X_{0}^{0}+Z_{0}^{0} \text {. }
$$

By the linearity of the derivative and since $\mathfrak{z}$ is a closed subspace of $\mathfrak{g}, \dot{Z}_{0}^{0}$ is the orthogonal projection of $\dot{a}_{2}(0)$ onto the center, so that

$$
\dot{a}_{2}(0)=\dot{X}_{0}^{0}+\dot{Z}_{0}^{0}
$$

is the orthogonal decomposition of $\dot{a}_{2}(0)$ into its central and noncentral components. For each $s$, let $X^{s}(t), Z^{s}(t)$ and $X^{\prime s}(t)$ denote the components of the geodesic flow as described in Subsection 3.5, generated by initial velocity $\alpha(s)$.

We now compute necessary and sufficient conditions for the curve $\dot{\alpha}(0)$ to satisfy Proposition 1.18 and thus generate a fixed point of $\Phi_{\tau *_{v_{p}}}$. These necessary and sufficient conditions are used throughout the proof of Theorem 3.1.

3.11 Theorem. Let $G$ be a two-step nilpotent Lie group with left invariant metric g. Let $\gamma=\exp (V+Z) \in G$. Let $\tilde{p}=x \tilde{q} a$, where $\tilde{q}^{-1} \gamma \tilde{q}=\exp \left(V+Z_{V}^{\perp}\right), x \in C(\gamma, G)$, and $a \in G$. Let $\alpha(s)=\left(\tilde{p} \exp \left(a_{1}(s)\right), a_{2}(s)\right) \in \tilde{\mathfrak{X}}$ such that $a_{1}(0)=0$ and $a_{2}(0)=v$. Let $a_{2}(s)=X_{0}^{s}+Z_{0}^{s}$ with $Z_{0}^{s} \in \mathfrak{z}$ and $X_{0}^{s} \in \mathfrak{v}$. Let $\exp \left(X^{s}(t)+Z^{s}(t)\right)$ be the geodesic through e with initial velocity $a_{2}(s)$. Then the geodesic flow of $(G, g)$ satisfies

$$
\tilde{\Phi}_{\tau *_{\tilde{p}}}(\dot{\alpha}(0))=L_{\gamma *} \dot{\alpha}(0)
$$

if and only if

(1) $X^{0}(\tau)=V$,

(2) $Z^{0}(\tau)=Z_{V}^{\perp}+[V, \log a]$

(3) $X^{\prime 0}(\tau)+Z_{0}^{0}=v$,

(4) $\dot{X}^{0}(\tau)=0$

(5) $\dot{Z}^{0}(\tau)=\left[V, \dot{a}_{1}(0)\right]$, and

(6) $\dot{X^{\prime} 0}(\tau)=\dot{X}_{0}^{0}$.

Recall that a prime denotes derivation with respect to the $t$ or $r$ variable and a dot denotes derivation with respect to the $s$ variable. 
Proof of Theorem 3.11. Let

$$
\beta(s)=\left(\tilde{\Phi}_{\tau} \circ \alpha\right)(s)
$$

Define $b_{1}(s)$ and $b_{2}(s)$ by

$$
\beta(s)=\left(\tilde{\Phi}_{\tau} \circ \alpha\right)(s)=\gamma \tilde{p}\left(\exp \left(b_{1}(s)\right), b_{2}(s)\right) .
$$

With this notation $\beta(0)=\gamma \cdot v_{\tilde{p}}=(\gamma \tilde{p}, v)$ if and only if

$$
b_{1}(0)=0 \text { and } b_{2}(0)=v \text {. }
$$

We now calculate the values of $b_{1}(s)$ and $b_{2}(s)$ in terms of $a_{1}(s)$ and $a_{2}(s)$.

$$
\begin{aligned}
\beta(s)= & \gamma \tilde{p} \cdot\left(\exp \left(b_{1}(s)\right), b_{2}(s)\right) \\
= & \left(\tilde{\Phi}_{\tau} \circ \alpha\right)(s) \\
= & \tilde{\Phi}_{\tau}\left(\tilde{p} \exp \left(a_{1}(s)\right), a_{2}(s)\right) \\
= & \tilde{p} \exp \left(a_{1}(s)\right) \cdot \tilde{\Phi}_{\tau}\left(e, a_{2}(s)\right) \\
= & \tilde{p} \exp \left(a_{1}(s)\right) \cdot\left(\exp \left(X^{s}(\tau)+Z^{s}(\tau)\right), X^{\prime s}(\tau)+Z_{0}^{s}\right) \\
= & x \tilde{q} a\left(a^{-1} \tilde{q}^{-1} \gamma \tilde{q} a\right) \\
& \cdot\left(\left(a^{-1} \tilde{q}^{-1} \gamma \tilde{q} a\right)^{-1} \exp \left(a_{1}(s)\right) \exp \left(X^{s}(\tau)+Z^{s}(\tau)\right), X^{\prime s}(\tau)+Z_{0}^{s}\right) \\
= & \gamma \tilde{p}\left(\exp \left(-V-Z_{V}^{\perp}-[V, \log a]\right) \exp \left(a_{1}(s)\right) \exp \left(X^{s}(\tau)+Z^{s}(\tau)\right), X^{\prime s}(\tau)+Z_{0}^{s}\right) .
\end{aligned}
$$

Thus by (2.1),

$$
b_{1}(s)=a_{1}(s)+X^{s}(\tau)-V+Z^{s}(\tau)-Z_{V}^{\perp}+\frac{1}{2}\left[a_{1}(s)-V, X^{s}(\tau)+V\right]-[V, \log a]
$$

and

$$
b_{2}(s)=X^{\prime s}(\tau)+Z_{0}^{s}
$$

Note that since $a_{1}(0)=0$,

$$
b_{1}(0)=0 \text { if and only if } X^{0}(\tau)=V \text { and } Z^{0}(\tau)=Z_{V}^{\perp}+[V, \log a],
$$

and

$$
b_{2}(0)=v \text { if and only if } v=X^{\prime 0}(\tau)+Z_{0}^{0} .
$$

In this case, since $\exp _{* \mid e}=\mathrm{Id}$,

$$
\tilde{\Phi}_{\tau *_{\tilde{p}}}(\dot{\alpha}(0))=\dot{\beta}(0)=\left(\dot{b}_{1}(0), \dot{b}_{2}(0)\right)_{\gamma \cdot v_{\tilde{p}}} .
$$

One easily calculates that

$$
\dot{b}_{1}(0)=\dot{a}_{1}(0)+\dot{X}^{0}(\tau)+\dot{Z}^{0}(\tau)+\left[\dot{a}_{1}(0)+\frac{1}{2} \dot{X}^{0}(\tau), V\right]
$$

and

$$
\dot{b}_{2}(0)=\dot{X}^{\prime 0}(\tau)+\dot{Z}_{0}^{0} .
$$

Thus $\dot{b}_{1}(0)=\dot{a}_{1}(0)$ if and only if $\dot{X}^{0}(\tau)+\dot{Z}^{0}(\tau)+\left[\dot{a}_{1}(0)+\frac{1}{2} \dot{X}^{0}(\tau), V\right]=0$, which since $\mathfrak{v}$ and $\mathfrak{z}$ are closed, implies $\dot{X}^{0}(\tau)=0$ and $\dot{Z}^{0}(\tau)=\left[V, \dot{a}_{1}(0)\right]$. Likewise $\dot{b}_{2}(0)=$ $\dot{a}_{2}(0)$ if and only if $\dot{X}^{\prime} 0(\tau)=\dot{X}_{0}^{0}$.

We have proved Theorem 3.11. 
We are now ready to prove the main theorem of the paper.

Proof of Theorem 3.1. Let $(\Gamma \backslash G, g)$ be a two-step nilmanifold.

We first show that if $\gamma=\exp (V+Z) \in \Gamma$ and $\vartheta_{m}\left(Z_{V}^{\perp}\right) \in 2 \pi \mathbb{Z}^{+}$for some $m=$ $1, \ldots, \mu\left(Z_{V}^{\perp}\right)$, then the nilmanifold $(\Gamma \backslash N, g)$ fails the Clean Intersection Hypothesis.

Let $\gamma=\exp (V+Z) \in \Gamma$. Let $\vartheta_{m}\left(Z_{V}^{\perp}\right) \in 2 \pi \mathbb{Z}^{+}$for some $m=1, \ldots, \mu\left(Z_{V}^{\perp}\right)$.

By Remark 3.9 , the value

$$
\tau=\left|V+Z_{V}^{\perp}\right|
$$

is always a period of $\gamma$. Let $\bar{V}=\frac{1}{\tau} V, \bar{Z}_{V}^{\perp}=\frac{1}{\tau} Z_{V}^{\perp}$, so $\bar{V}+\bar{Z}_{V}^{\perp}$ is a unit vector in $\mathfrak{g}$, and $\tau j\left(\bar{Z}_{V}^{\perp}\right)=j\left(Z_{V}^{\perp}\right)$. By Theorem 3.8 and Remarks 3.9, we have

$$
F\left(\tau, c_{\gamma}\right)=\pi\left(C(\gamma, G) \tilde{q} \times\left\{\bar{V}+\bar{Z}_{V}^{\perp}\right\}\right),
$$

where

$$
\tilde{q}^{-1} \gamma \tilde{q}=\exp \left(V+Z_{V}^{\perp}\right)
$$

and $C(\gamma, G)$ is the centralizer of $\gamma$ in $G$. That is, for all $v_{p} \in F\left(\tau, c_{\gamma}\right), v=\bar{V}+\bar{Z}_{V}^{\perp}$ and $p \in \pi(C(\gamma, G) \tilde{q})$, so that

$$
T_{v_{p}} F\left(\tau, c_{\gamma}\right)=T_{p}(\pi(C(\gamma, G) \tilde{q})) \times\{0\} .
$$

Let

$$
\tilde{p}=x \tilde{q}
$$

for some $x \in C(\gamma, G)$ and let $p=\pi(\tilde{p})$. So in the notation of 3.10, $a=e$. Note that $\tilde{p} \exp \left(V+Z_{V}^{\perp}\right)=\tilde{p} \tilde{q}^{-1} \gamma \tilde{q}=\gamma \tilde{p}$, since $x \in C(\gamma, G)$, let $v_{\tilde{p}}=\left(\tilde{p}, \bar{V}+\bar{Z}_{V}^{\perp}\right)$, so that $\pi_{*}\left(v_{\tilde{p}}\right)=v_{p} \in F\left(\tau, c_{\gamma}\right)$.

Let $\xi \in W_{m}\left(Z_{V}^{\perp}\right),|\xi|=1$, let

$$
X_{0}^{s}=\sin (s) \xi+\cos (s) \bar{V} \text { and } Z_{0}^{s}=\cos (s) \bar{Z}_{V}^{\perp},
$$

and let

$$
a_{2}(s)=X_{0}^{s}+Z_{0}^{s} .
$$

Note that $\xi \perp\left(\bar{V}+\bar{Z}_{V}^{\perp}\right)$, since $\bar{V} \in \operatorname{ker} j\left(\bar{Z}_{V}^{\perp}\right)$ and $\vartheta_{m}\left(Z_{V}^{\perp}\right)>0$, so $\left|X_{0}^{s}+Z_{0}^{s}\right|=1$. Also note that, in the notation of 3.3,

$$
\begin{aligned}
& X_{1}^{s}=\cos (s) \bar{V} \subset \operatorname{ker} j\left(Z_{0}^{s}\right)=\operatorname{ker} j\left(Z_{V}^{\perp}\right), \\
& X_{2}^{s}=\sin (s) \xi \subset W_{m}\left(Z_{0}^{s}\right)=W_{m}\left(Z_{V}^{\perp}\right),
\end{aligned}
$$

so that $\dot{a}_{2}(0)=\xi$.

Let $\alpha(s)=\left(\tilde{p} \exp \left(a_{1}(s)\right), X_{0}^{s}+Z_{0}^{s}\right) \in G \times S^{n-1} \cong \tilde{\mathfrak{X}}$, where $a_{1}(s)$ is a curve in $\mathfrak{g}$ with $a_{1}(0)=0$ and with derivative $\dot{a}_{1}(0)$ that will be specified later. Note that $\alpha(0)=\left(\tilde{p}, \bar{V}+\bar{Z}_{V}^{\perp}\right)=v_{\tilde{p}}$, and $\dot{\alpha}(0)=\left(\dot{a}_{1}(0), \xi\right)_{v_{\tilde{p}}} \in T_{v_{\tilde{p}}} \tilde{\mathfrak{X}}$, so that

$$
\pi_{*}\left(\dot{\alpha}(0)_{v_{\tilde{p}}}\right)=\left(\dot{a}_{1}(0), \xi\right)_{v_{p}} \in T_{v_{p}} \mathfrak{X}
$$

but clearly

since $\xi \neq 0$.

$$
\left(\dot{a}_{1}(0), \xi\right)_{v_{p}} \notin T_{v_{p}} F\left(\tau, c_{\gamma}\right),
$$

To show that $\left(\dot{a}_{1}(0), \xi\right)_{v_{p}}$ is a fixed point of $\Phi_{\tau_{*_{v}}}$, we must show that $\left(\dot{a}_{1}(0), \xi\right)_{v_{\tilde{p}}}$ satisfies conditions (1) through (6) of Theorem 3.11.

Note that by Definitions 2.4 and Notation $3.2, j\left(Z_{V}^{\perp}\right) \xi \in W_{m}\left(Z_{V}^{\perp}\right)$, and $j\left(Z_{V}^{\perp}\right)^{2} \xi$ $=-\vartheta_{m}\left(Z_{V}^{\perp}\right)^{2} \xi$. Using the fact that $\vartheta_{m}\left(Z_{V}^{\perp}\right) \in 2 \pi \mathbb{Z}^{+}$and by Notation 3.2, one easily calculates that

$$
e^{j\left(Z_{V}^{\perp}\right)}{ }_{\mid W_{m}\left(Z_{V}^{\perp}\right)}=\operatorname{Id}_{\mid W_{m}\left(Z_{V}^{\perp}\right)} .
$$


Since $j\left(\bar{Z}_{V}^{\perp}\right)^{-1} \xi \in W_{m}\left(Z_{V}^{\perp}\right)$,

$$
\left(e^{j\left(Z_{V}^{\perp}\right)}-\mathrm{Id}\right) j\left(\bar{Z}_{V}^{\perp}\right)^{-1} \xi=0 .
$$

Since $j\left(Z_{0}^{s}\right)=\cos (s) j\left(\bar{Z}_{V}^{\perp}\right)$,

$$
j\left(Z_{0}^{s}\right)^{-1}=\sec (s) j\left(\bar{Z}_{V}^{\perp}\right)^{-1} .
$$

We now calculate from Subsection 3.5 that

$$
\begin{aligned}
X^{s}(t) & =t X_{1}^{s}+\left(e^{t j\left(Z_{0}^{s}\right)}-\mathrm{Id}\right) j\left(Z_{0}^{s}\right)^{-1} X_{2}^{s} \\
& =t \cos (s) \bar{V}+\tan (s)\left(e^{\cos (s) t j\left(\bar{Z}_{\bar{V}}^{\perp}\right)}-\mathrm{Id}\right) j\left(\bar{Z}_{V}^{\perp}\right)^{-1} \xi,
\end{aligned}
$$

so $X^{0}(t)=t \bar{V}$ and $X^{0}(\tau)=V$; therefore, condition (1) of Theorem 3.11 is satisfied. Also by Subsection 3.5,

$$
X^{\prime s}(t)=X_{1}^{s}+e^{t j\left(Z_{0}^{s}\right)} X_{2}^{s}=\cos (s) \bar{V}+\sin (s) e^{\cos (s) t j\left(\bar{Z}_{\bar{V}}^{\perp}\right)} \xi,
$$

so $X^{\prime 0}(t)=\bar{V}$ and condition (3) of Theorem 3.11 is satisfied. Finally from Subsection 3.5 ,

so

$$
Z^{s}(\tau)=\int_{0}^{\tau}\left(Z_{0}^{s}+\frac{1}{2}\left[X^{s}(t), X^{\prime s}(t)\right]\right) d t
$$

$$
Z^{0}(\tau)=\int_{0}^{\tau}\left(\bar{Z}_{V}^{\perp}+\frac{1}{2}[t \bar{V}, \bar{V}]\right) d t=Z_{V}^{\perp} .
$$

Thus condition (2) of Theorem 3.11 is satisfied, since $a=e$ and $\log e=0$.

Now

$$
\begin{aligned}
\dot{X}^{s}(t)= & \frac{d}{d s}\left\{\cos (s) t \bar{V}+\tan (s)\left(e^{\cos (s) t j\left(\bar{Z}_{V}^{\perp}\right)}-\mathrm{Id}\right) j\left(\bar{Z}_{V}^{\perp}\right)^{-1} \xi\right\} \\
=- & \sin (s) t \bar{V}+\sec ^{2}(s)\left\{\left(e^{\cos (s) t j\left(\bar{Z}_{V}^{\perp}\right)}-\mathrm{Id}\right) j\left(\bar{Z}_{V}^{\perp}\right)^{-1} \xi\right\} \\
& \quad+\tan (s) \frac{d}{d s}\left\{\left(e^{\cos (s) t j\left(\bar{Z}_{V}^{\perp}\right)}-\mathrm{Id}\right) j\left(\bar{Z}_{V}^{\perp}\right)^{-1} \xi\right\}
\end{aligned}
$$

and so

$$
\dot{X}^{0}(\tau)=\left(e^{j\left(Z_{V}^{\perp}\right)}-\mathrm{Id}\right) j\left(\bar{Z}_{V}^{\perp}\right)^{-1} \xi=0
$$

and condition (4) of Theorem 3.11 is satisfied.

From above,

$$
\dot{X^{\prime} s}(t)=-\sin (s) \bar{V}+\cos (s) e^{\cos (s) t j\left(\bar{Z}_{V}^{\perp}\right)} \xi+\sin (s) \frac{d}{d s}\left\{e^{\cos (s) t j\left(\bar{Z}_{V}^{\perp}\right)} \xi\right\}
$$

and so

$$
\dot{X^{\prime} 0}(\tau)=e^{j\left(Z_{V}^{\perp}\right)} \xi=\xi=\dot{X}_{0}^{0}
$$

and condition (6) of Theorem 3.11 is satisfied.

Finally, from Subsection 3.5

$$
\frac{d}{d s} Z^{s}(\tau)=\frac{d}{d s} \int_{0}^{\tau}\left(Z_{0}^{s}+\frac{1}{2}\left[X^{s}(t), X^{\prime s}(t)\right]\right) d t .
$$

Since everything in sight is analytic in both $s$ and $t$ and bounded with a bounded derivative in $s$ near 0 , and since we are integrating over a finite, closed interval, we can use uniform convergence to bring the derivative $\frac{d}{d s}$ under the integral sign. 
Using the linearity of the Lie bracket in each component and the product rule, we thus have

$$
\begin{aligned}
\frac{d}{d s} Z^{s}(\tau) & =\int_{0}^{\tau} \frac{d}{d s}\left(Z_{0}^{s}+\frac{1}{2}\left[X^{s}(t), X^{\prime s}(t)\right]\right) d t \\
& =\int_{0}^{\tau}\left(-\sin (s) \bar{Z}+\frac{1}{2}\left[\frac{d}{d s} X^{s}(t), X^{\prime s}(t)\right]+\frac{1}{2}\left[X^{s}(t), \frac{d}{d s} X^{\prime s}(t)\right]\right) d t
\end{aligned}
$$

and so

$$
\dot{Z}^{0}(\tau)=\frac{1}{2} \int_{0}^{\tau}\left(\left[\dot{X}^{0}(t), \bar{V}\right]+\left[t \bar{V}, \dot{X}^{\prime 0}(t)\right]\right) d t
$$

Since $[V, \mathfrak{g}]$ is a closed subspace of $\mathfrak{z}$, we conclude that $\dot{Z}^{0}(\tau) \subset[V, \mathfrak{g}]$.

We now choose $a_{1}(s)$ so that

$$
\left[V, \dot{a}_{1}(0)\right]=\dot{Z}^{0}(\tau)
$$

With this choice of $a_{1}(s)$, condition (5) of Theorem 3.11 is satisfied.

Since $\alpha(s)=\left(\tilde{p} \exp \left(a_{1}(s)\right), a_{2}(s)\right)$ satisfies conditions (1) through (6) of Theorem 3.11 , we conclude that

$$
\tilde{\Phi}_{\tau * v_{\tilde{p}}}\left((\dot{a}(0), \xi)_{v_{\tilde{p}}}\right)=(\dot{a}(0), \xi)_{\gamma \cdot v_{\tilde{p}}} .
$$

As $v_{\tilde{p}}$ and $\gamma \cdot v_{\tilde{p}}$ are identified under $\pi: \mathfrak{X} \rightarrow \tilde{X}$, we see that $\left(\dot{a}_{1}(0), \xi\right)_{v_{p}} \in T_{v_{p}} \mathfrak{X}$ is a fixed point of $\Phi_{\tau_{*_{v}}}$, but $\left(\dot{a}_{1}(0), \xi\right)_{v_{p}}$ is not in $T_{v_{p}} F\left(\tau, c_{\gamma}\right)$.

We have proved that if $\gamma=\exp (V+Z) \in \Gamma$ and $\vartheta_{m}\left(Z_{V}^{\perp}\right) \in 2 \pi \mathbb{Z}^{+}$, then $\Gamma \backslash G$ does not satisfy the Clean Intersection Hypothesis.

We now prove that the only "unclean" lengths $\tau$ are those associated with a $\gamma=\exp (V+Z) \in \Gamma$ such that $\tau=\left|V+Z_{V}^{\perp}\right|$ and $\vartheta_{m}\left(Z_{V}^{\perp}\right) \in 2 \pi \mathbb{Z}^{+}$for some $m \in\left\{1, \ldots, \mu\left(Z_{V}^{\perp}\right)\right\}$.

To do this, let $\alpha(s)=\left(\tilde{p} \exp \left(a_{1}(s), a_{2}(s)\right) \in \tilde{\mathfrak{X}}\right.$ with $a_{1}(0)=0$ and $a_{2}(0)=v$ where $v_{\tilde{p}} \in \tilde{F}(\tau, \gamma)$. Assume that

$$
\tilde{\Phi}_{\tau *_{\tilde{p}}}(\dot{\alpha}(0))=L_{\gamma *} \dot{\alpha}(0),
$$

i.e., by Proposition 1.18, assume that $\pi_{*}(\dot{\alpha}(0))$ is a fixed point of $\Phi_{\tau *_{v_{p}}}$ for $v_{p} \in$ $F\left(\tau, c_{\gamma}\right)$. We use the fact that $\dot{\alpha}(0)$ satisfies conditions (1) through (6) of Theorem 3.11 to show that either $\tau=\left|V+Z_{V}^{\perp}\right|$ and $\vartheta_{m}\left(Z_{V}^{\perp}\right) \in 2 \pi \mathbb{Z}^{+}$for some $m \in\left\{1, \ldots, \mu\left(Z_{V}^{\perp}\right)\right\}$, or else $\dot{\alpha}(0)$ is tangent to $\tilde{F}(\tau, \gamma)$ and hence $\pi_{*}(\dot{\alpha}(0))$ is tangent to $F\left(\tau, c_{\gamma}\right)$.

Because we will be taking limits and derivatives of vector curves $X_{s}+Z_{s}$ in $\mathfrak{g}$, and because the number of distinct eigenvalues of $j\left(Z_{s}\right)$ can vary with $s$, we will need the following definitions and properties.

3.12 Definition. Let $(\mathfrak{g},\langle\rangle$,$) be a two-step nilpotent metric Lie algebra. Define$ $\mathcal{U}=\{Z \in \mathfrak{z}$ : there exists an open neighborhood $\mathcal{O}$ of $Z$ such that $\mu$ is constant on $\mathcal{O}\}$. We call $\mathcal{U}$ the simple subdomain of $\mathfrak{z}$.

3.13 Proposition ([GtM1 Prop. 1.19]). Let $(\mathfrak{g},\langle\rangle$,$) be a two-step nilpotent$ metric Lie algebra. Then the following hold:

(1) The simple subdomain $\mathcal{U}$ is open and dense in $\mathfrak{z}$.

(2) The function $\mu(Z)$ is constant on $\mathcal{U}$.

(3) The function $\vartheta_{m}: \mathcal{U} \rightarrow \mathbb{R}$ is smooth on $\mathcal{U}-\{0\}$ for $m=1, \ldots, \mu(Z)$.

(4) If $Z$ is a limit point of $\mathcal{U}$, then $\mu(Z) \leq \mu(\mathcal{U})$. 
First assume that $Z_{0} \in \mathcal{U}$. We may then assume that the number of distinct eigenvalues of $j\left(Z_{s}\right)$ is constant for $s$ in a neighborhood of 0 . By $[\mathrm{A}]$, if $Z_{0}^{s}$ and $X_{0}^{s}$ are analytic in $s$, we know that the invariant subspace $W_{m}\left(Z_{s}\right)$, the eigenvalue curves $\vartheta_{m}\left(Z_{0}^{s}\right)$, and the eigenvector curves $X_{1}^{s}, X_{2}^{s}$, and $\xi_{m}^{s}$ as defined in (3.2) and (3.3) are analytic in $s, m=1, \ldots, \mu$. Thus all of the limits and derivatives exist below.

If $Z_{0} \notin \mathcal{U}$, then we must be more precise. As $\mathcal{U}$ is dense in $\mathfrak{z}, Z_{0}$ is a limit point of $\mathcal{U}$. By Proposition 3.13 and continuity of the set of (unordered) eigenvalues [Kt, §II.5], two of the eigenvalue curves must approach each other as $Z$ approaches $Z_{0} \in \mathfrak{z}-\mathcal{U}$ and the counting function $\mu$ has a discontinuity at $Z_{0}$. We proceed as though exactly two eigenvalue curves $\vartheta_{m^{\prime}}$ and $\vartheta_{m^{\prime \prime}}$ intersect at $Z_{0}$; the statements generalize in the obvious manner in the case that more than two eigenvalue curves intersect at $Z_{0}$. As $\mathcal{U}$ is dense and open in $\mathfrak{z}$, there exist $Z_{s} \rightarrow Z_{0}, Z_{s} \in \mathcal{U}$, such that $\frac{d}{d s \mid 0} Z_{s}$ takes any desired value, and such that as $s \rightarrow 0$,

$$
\lim _{s \rightarrow 0} \vartheta_{m^{\prime}}\left(Z_{s}\right)=\vartheta_{m}\left(Z_{0}\right) \text { and } \lim _{s \rightarrow 0} \vartheta_{m^{\prime \prime}}\left(Z_{s}\right)=\vartheta_{m}\left(Z_{0}\right)
$$

We may assume that the curves $\vartheta_{m^{\prime}}\left(Z_{s}\right)$ and $\vartheta_{m^{\prime \prime}}\left(Z_{s}\right)$ are analytic (in $s$ ) in what follows.

Note that if we write $X_{0}^{s}=X_{1}^{s}+X_{2}^{s}$, where $X_{1}^{s} \in \operatorname{ker} j\left(Z_{s}\right)$ and $X_{2}^{s} \perp \operatorname{ker} j\left(Z_{s}\right)$, and if we write $X_{2}^{s}=\sum_{m} \xi_{m}^{s}$, then the subscript $m$ might depend on $s$ in the limit. If this is the case, we view $W_{m}\left(Z_{0}\right)$ as the sum of its refined invariant subspaces, which we now define.

For sufficiently small positive values of $s, j\left(Z_{s}\right)$ has invariant subspaces $W_{m^{\prime}}\left(Z_{s}\right)$ and $W_{m^{\prime \prime}}\left(Z_{s}\right)$, respectively. By $\left[\mathrm{A}\right.$, Theorem 4.16], since $j\left(Z_{s}\right) \rightarrow j\left(Z_{0}\right)$ and skewsymmetry holds, we may define

$$
W^{\prime}\left(Z_{0}\right)=\lim _{s \rightarrow 0} W_{m^{\prime}}\left(Z_{s}\right) \quad \text { and } \quad W^{\prime \prime}\left(Z_{0}\right)=\lim _{s \rightarrow 0} W_{m^{\prime \prime}}\left(Z_{s}\right) .
$$

Note that since $W_{m^{\prime}}\left(Z_{s}\right)$ and $W_{m^{\prime \prime}}\left(Z_{s}\right)$ are orthogonal, invariant subspaces of $j\left(Z_{s}\right)$ for all $s$, their limit spaces $W^{\prime}\left(Z_{0}\right), W^{\prime \prime}\left(Z_{0}\right)$ are orthogonal, invariant subspaces of $j\left(Z_{0}\right)$, and

$$
W_{m}\left(Z_{0}\right)=W^{\prime}\left(Z_{0}\right) \oplus W^{\prime \prime}\left(Z_{0}\right)
$$

We refer to $W^{\prime}\left(Z_{0}\right), W^{\prime \prime}\left(Z_{0}\right)$ as refined invariant subspaces of $j\left(Z_{0}\right)$.

3.14 Proposition. Let $(\mathfrak{g},\langle\rangle$,$) be a two-step nilpotent metric Lie algebra and let$ $Z_{0} \in \mathfrak{z}-\mathcal{U}$. Let $Z_{s}$ be an analytic (in $s$ ) curve in $\mathcal{U}$ such that $\lim _{s \rightarrow 0} Z_{s}=Z_{0}$, $\lim _{s \rightarrow 0} \vartheta_{m^{\prime}}\left(Z_{s}\right)=\vartheta_{m}\left(Z_{0}\right)$ and $\lim _{s \rightarrow 0} \vartheta_{m^{\prime \prime}}\left(Z_{s}\right)=\vartheta_{m}\left(Z_{0}\right), m^{\prime} \neq m^{\prime \prime}$. With notation as above, let $\xi_{m^{\prime}}^{s} \in W_{m^{\prime}}\left(Z_{s}\right)$ and $\xi_{m^{\prime \prime}}^{s} \in W_{m^{\prime \prime}}\left(Z_{s}\right)$ such that $\lim _{s \rightarrow 0} \xi_{m^{\prime}}^{s}=\xi^{\prime} \in$ $W^{\prime}\left(Z_{0}\right)$ and $\lim _{s \rightarrow 0} \xi_{m^{\prime \prime}}^{s}=\xi^{\prime \prime} \in W^{\prime \prime}\left(Z_{0}\right)$. Then

$$
\left[\xi^{\prime}+\xi^{\prime \prime}, j\left(Z_{0}\right)\left(\xi^{\prime}+\xi^{\prime \prime}\right)\right]=\left[\xi^{\prime}, j\left(Z_{0}\right) \xi^{\prime}\right]+\left[\xi^{\prime \prime}, j(\zeta) \xi^{\prime \prime}\right] .
$$

In particular,

$$
\left[\xi^{\prime}, j\left(Z_{0}\right) \xi^{\prime \prime}\right]+\left[\xi^{\prime \prime}, j\left(Z_{0}\right) \xi^{\prime}\right]=0
$$

and the mapping $K: \mathfrak{v} \rightarrow \mathfrak{z}$ defined in Definitions 3.4 is continuous.

Proof of Proposition 3.14. This result is contained in the proof of Lemma 4.6 in GtM1.

Thus, by passing to refined invariant subspaces when necessary, we deduce that all of the derivatives exist below, even in the case that $Z_{0} \notin \mathcal{U}$, and that the limiting values are as given. For most of the proof, we do not separate the cases $Z_{0} \in \mathcal{U}$ and 
$Z_{0} \notin \mathcal{U}$ in what follows. The reader should just consider the curves $\xi_{m}^{s}$ contained in refined invariant subspaces rather than the invariant subspaces $W_{m}\left(Z_{0}^{s}\right)$ when necessary. We must, however, be careful in the last steps of Case II below.

Another technical detail in the remainder of this proof is the possibility that $\lim _{s \rightarrow 0} \vartheta_{m}\left(Z_{0}^{s}\right)=0$ for some $m$ such that $\xi_{m}^{s} \not \equiv 0$. To account for this, we write

$$
\left\{1, \ldots, \mu\left(Z_{0}\right)\right\}=N \cup M,
$$

where $N$ is defined as the subset of $\{1, \ldots, \mu\}$ such that, for $n \in N, \lim _{s \rightarrow 0} \vartheta_{n}\left(Z_{0}^{s}\right)=$ 0 and $\vartheta_{n}\left(Z_{0}^{s}\right)>0$ for all $s \neq 0$. Define $M$ as the subset of $\{1, \ldots, \mu\}$ such that, for $m \in M, \lim _{s \rightarrow 0} \vartheta_{m}\left(Z_{0}^{s}\right) \neq 0$. Thus $X_{2}^{s}=\sum_{m \in M} \xi_{m}^{s}+\sum_{n \in N} \xi_{n}^{s}$. Note that if $\vartheta_{1}\left(Z_{0}^{s}\right)=0$ for all $s$, then $\xi_{1}^{s}=0$ for all $s$, and the vector component corresponding to $\vartheta_{1}\left(Z_{0}^{s}\right)$ is included in $X_{1}^{s}$ and not in $X_{2}^{s}$.

Because of this possibility, the notations $X_{1}^{0}$ and $X_{2}^{0}$ are ambiguous. We use the convention that $a_{2}(0)=X_{1}+X_{2}+Z_{0}$ as in Notation 3.3, while $X_{i}^{0}=\lim _{s \rightarrow 0} X_{i}^{s}$, $i=1,2$, and $Z_{0}^{0}=\lim _{s \rightarrow 0} Z_{0}^{s}$. Thus $Z_{0}^{0}=Z_{0}$ but

$$
X_{1}=X_{1}^{0}+\sum_{n \in N} \xi_{n}^{0}
$$

and

$$
X_{2}=X_{2}^{0}-\sum_{n \in N} \xi_{n}^{0}=\sum_{m \in M} \xi_{m}^{0}
$$

where we define $\xi_{m}^{0}=\lim _{s \rightarrow 0} \xi_{m}^{s}, J_{s}=j\left(Z_{0}^{s}\right)$, and $\vartheta_{m}^{s}=\vartheta_{m}\left(Z_{0}^{s}\right)$.

We now proceed with the proof of the necessary condition of Theorem 3.1.

Let $\alpha(s)=\left(\tilde{p} \exp \left(a_{1}(s)\right), a_{2}(s)\right)$ be a curve in $\tilde{\mathfrak{X}}$ such that

$$
\tilde{\Phi}_{\tau *_{\tilde{p}}}(\dot{\alpha}(0))=\tilde{\Phi}_{\tau *_{\tilde{p}}}\left(\left(\dot{a}_{1}(0), \dot{a}_{2}(0)\right)_{v_{\tilde{p}}}\right)=\left(\dot{a}_{1}(0), \dot{a}_{2}(0)\right)_{\gamma \cdot v_{\tilde{p}}},
$$

with $a_{1}(0)=0, a_{2}(0)=v=X_{1}+X_{2}+Z_{0}$, and $v_{p} \in F\left(\tau, c_{\gamma}\right)$. Now $a_{2}(s)=$ $X_{1}^{s}+X_{2}^{s}+Z_{0}^{s}$, where $Z_{0}^{s} \in \mathfrak{z}, X_{1}^{s}+X_{2}^{s} \perp \mathfrak{z}, X_{1}^{s} \in \operatorname{ker} j\left(Z_{0}^{s}\right)$ and $X_{2}^{s} \perp \operatorname{ker} j\left(Z_{0}^{s}\right)$. Also, $X_{2}^{s}=\sum_{n \in N} \xi_{n}^{s}+\sum_{m \in M} \xi_{m}^{s}$, where $\xi_{m}^{s} \in W_{m}\left(Z_{0}^{s}\right)$ and $\xi_{n}^{s} \in W_{n}\left(Z_{0}^{s}\right)$ as above.

The geodesic with initial velocity $\alpha(s)$ can be written as

$$
\tilde{p} \exp \left(a_{1}(s)\right) \exp \left(X^{s}(t)+Z^{s}(t)\right),
$$

with $a_{1}(0)=0$. Using Subsection 3.5, we see that the values for $X^{s}(r)$ and $Z^{s}(r)$ are as follows:

$$
\begin{aligned}
X^{s}(r)= & r X_{1}^{s}+\left(e^{r J_{s}}-\mathrm{Id}\right)\left(J_{s}^{-1} X_{2}^{s}\right) \\
= & r X_{1}^{s}+\sum_{m \in M} \frac{1-\cos \left(r \vartheta_{m}^{s}\right)}{\vartheta_{m}^{s}}\left(\frac{1}{\vartheta_{m}^{s}} J_{s} \xi_{m}^{s}\right)+\sum_{m \in M} \frac{\sin \left(r \vartheta_{m}^{s}\right)}{\vartheta_{m}^{s}} \xi_{m}^{s} \\
& \quad+\sum_{n \in N} \frac{1-\cos \left(r \vartheta_{n}^{s}\right)}{\vartheta_{n}^{s}}\left(\frac{1}{\vartheta_{n}^{s}} J_{s} \xi_{n}^{s}\right)+\sum_{n \in N} \frac{\sin \left(r \vartheta_{n}^{s}\right)}{\vartheta_{n}^{s}} \xi_{n}^{s} \\
X^{\prime s}(r)= & X_{1}^{s}+e^{r J_{s}} X_{2}^{s} \\
= & X_{1}^{s}+\sum_{m \in M} \sin \left(r \vartheta_{m}^{s}\right)\left(\frac{1}{\vartheta_{m}^{s}} J_{s} \xi_{m}^{s}\right)+\sum_{m \in M} \cos \left(r \vartheta_{m}^{s}\right) \xi_{m}^{s} \\
& \quad+\sum_{n \in N} \sin \left(r \vartheta_{n}^{s}\right)\left(\frac{1}{\vartheta_{n}^{s}} J_{s} \xi_{n}^{s}\right)+\sum_{n \in N} \cos \left(r \vartheta_{n}^{s}\right) \xi_{n}^{s} \\
Z^{s}(\tau)= & \int_{0}^{\tau}\left(Z_{0}^{s}+\frac{1}{2}\left[X^{s}(r), X^{\prime s}(r)\right]\right) d r .
\end{aligned}
$$


Taking the derivative with respect to $s$ of $X^{s}(r)$ and evaluating at $s=0$, we have

$$
\begin{aligned}
\dot{X}^{0}(r) & =r \dot{X}_{1}^{0}+\sum_{m} \dot{\vartheta}_{m}^{0}\left(\frac{r \vartheta_{m}^{0} \sin \left(r \vartheta_{m}^{0}\right)+\cos \left(r \vartheta_{m}^{0}\right)-1}{\vartheta_{m}^{0}{ }^{2}}\right) \frac{1}{\vartheta_{m}^{0}} J_{0} \xi_{m}^{0} \\
& +\sum_{m} \dot{\vartheta}_{m}^{0}\left(\frac{r \vartheta_{m}^{0} \cos \left(r \vartheta_{m}^{0}\right)-\sin \left(r \vartheta_{m}^{0}\right)}{\vartheta_{m}^{0}{ }^{2}}\right) \xi_{m}^{0}+\left.\sum_{m} \frac{1-\cos \left(r \vartheta_{m}^{0}\right)}{\vartheta_{m}^{0}} \frac{d}{d s}\right|_{0}\left(\frac{1}{\vartheta_{m}^{s}} J_{s} \xi_{m}^{s}\right) \\
& +\sum_{m} \frac{\sin \left(r \vartheta_{m}^{0}\right)}{\vartheta_{m}^{0}} \dot{\xi}_{m}^{0}+\sum_{n} \dot{\vartheta}_{n}^{0}\left(\frac{r \vartheta_{n}^{0} \sin \left(r \vartheta_{n}^{0}\right)+\cos \left(r \vartheta_{n}^{0}\right)-1}{\vartheta_{n}^{0}}\right) \frac{1}{\vartheta_{n}^{0}} J_{0} \xi_{n}^{0} \\
& +\sum_{n} \dot{\vartheta}_{n}^{0}\left(\frac{r \vartheta_{n}^{0} \cos \left(r \vartheta_{n}^{0}\right)-\sin \left(r \vartheta_{n}^{0}\right)}{\vartheta_{n}^{0}{ }^{2}}\right) \xi_{n}^{0}+\left.\sum_{n} \frac{1-\cos \left(r \vartheta_{n}^{0}\right)}{\vartheta_{n}^{0}} \frac{d}{d s}\right|_{0}\left(\frac{1}{\vartheta_{n}^{s}} J_{s} \xi_{n}^{s}\right) \\
& +\sum_{n} \frac{\sin \left(r \vartheta_{n}^{0}\right)}{\vartheta_{n}^{0}} \dot{\xi}_{n}^{0} .
\end{aligned}
$$

Note that for $m \in M$, the values above are clearly well defined, since all of the values are analytic in $s$. For $n \in N$, observe that $\left|\frac{1}{\vartheta_{n}^{s}} J_{s} \xi_{n}^{s}\right|=\left|\xi_{n}^{s}\right|$ by using the definition of $\xi_{n}^{s},(3.2)$ and (3.3), so $\frac{1}{\vartheta_{n}^{s}} J_{s} \xi_{n}^{s}$ is likewise well defined with a continuous derivative in $s$. Observe that for $n \in N$ we are abusing notation slightly. For example, for $n \in N, \sin \left(r \vartheta_{n}^{0}\right) / \vartheta_{n}^{0}$ is technically not defined, while $\lim _{s \rightarrow 0} \sin \left(r \vartheta_{n}^{s}\right) / \vartheta_{n}^{s}$ exists and is well defined. We use the convention that for $n \in N$, when necessary, seemingly undefined values are defined as the limit as $s \rightarrow 0$ of nearby values. With this established, for $n \in N$,

$$
\begin{aligned}
& \left(\frac{r \vartheta_{n}^{0} \sin \left(r \vartheta_{n}^{0}\right)+\cos \left(r \vartheta_{n}^{0}\right)-1}{\vartheta_{n}^{0}}\right)=\frac{r^{2}}{2}, \quad\left(\frac{r \vartheta_{n}^{0} \cos \left(r \vartheta_{n}^{0}\right)-\sin \left(r \vartheta_{n}^{0}\right)}{\vartheta_{n}^{0}}\right)=0, \\
& \frac{\dot{\vartheta}_{n}^{0} J_{0} \xi_{n}^{0}}{\vartheta_{n}^{0}}=\left.\frac{d}{d s}\right|_{\left.\right|_{0}}\left(J_{s} \xi_{n}^{s}\right), \quad \frac{1-\cos \left(r \vartheta_{n}^{0}\right)}{\vartheta_{n}^{0}}=0, \quad \frac{\sin \left(r \vartheta_{n}^{0}\right)}{\vartheta_{n}^{0}}=r .
\end{aligned}
$$

We now have

$$
\begin{aligned}
\dot{X}^{0}(r) & =r\left(\dot{X}_{1}^{0}+\sum_{n \in N} \dot{\xi}_{n}^{0}\right)+\sum_{m \in M} \dot{\vartheta}_{m}^{0}\left(\frac{r \vartheta_{m}^{0} \sin \left(r \vartheta_{m}^{0}\right)+\cos \left(r \vartheta_{m}^{0}\right)-1}{\vartheta_{m}^{0}{ }^{2}}\right) \frac{1}{\vartheta_{m}^{0}} J_{0} \xi_{m}^{0} \\
& +\sum_{m \in M} \dot{\vartheta}_{m}^{0}\left(\frac{r \vartheta_{m}^{0} \cos \left(r \vartheta_{m}^{0}\right)-\sin \left(r \vartheta_{m}^{0}\right)}{\vartheta_{m}^{0}{ }^{2}}\right) \xi_{m}^{0} \\
(\text { () }) \quad & +\left.\sum_{m \in M} \frac{1-\cos \left(r \vartheta_{m}^{0}\right)}{\vartheta_{m}^{0}} \frac{d}{d s}\right|_{\left.\right|_{0}}\left(\frac{1}{\vartheta_{m}^{s}} J_{s} \xi_{m}^{s}\right) \\
& +\sum_{m \in M} \frac{\sin \left(r \vartheta_{m}^{0}\right)}{\vartheta_{m}^{0}} \dot{\xi}_{m}^{0}+\sum_{n \in N} \frac{r^{2}}{2} \frac{d}{d s}{ }_{\left.\right|_{0}}\left(J_{s} \xi_{n}^{s}\right) .
\end{aligned}
$$


A similar analysis yields

$$
\begin{aligned}
\dot{X}^{\prime 0}(r) & =\left(\dot{X}_{1}^{0}+\sum_{n \in N} \dot{\xi}_{n}^{0}\right)+\sum_{m \in M} r \dot{\vartheta}_{m}^{0} \cos \left(r \vartheta_{m}^{0}\right) \frac{1}{\vartheta_{m}^{0}} J_{0} \xi_{m}^{0} \\
& -\sum_{m \in M} r \dot{\vartheta}_{m}^{0} \sin \left(r \vartheta_{m}^{0}\right) \xi_{m}^{0}+\left.\sum_{m \in M} \sin \left(r \vartheta_{m}^{0}\right) \frac{d}{d s}\right|_{\left.\right|_{0}}\left(\frac{1}{\vartheta_{m}^{s}} J_{s} \xi_{m}^{s}\right) \\
& +\sum_{m \in M} \cos \left(r \vartheta_{m}^{0}\right) \dot{\xi}_{m}^{0}+\left.\sum_{n \in N} r \frac{d}{d s}\right|_{\left.\right|_{0}}\left(J_{s} \xi_{n}^{s}\right) .
\end{aligned}
$$

We now consider two special cases, and use the fact that $\dot{\alpha}(0)$ must satisfy conditions (1) through (6) of Theorem 3.11.

Case I: $\tau=\left|V+Z_{V}^{\perp}\right|$.

If $\vartheta_{m}\left(Z_{V}^{\perp}\right) \in 2 \pi \mathbb{Z}^{+}$for some $m \in\left\{1, \ldots, \mu\left(Z_{V}^{\perp}\right)\right\}$, then we are done. We thus assume that $\vartheta_{m}\left(Z_{V}^{\perp}\right) \notin 2 \pi \mathbb{Z}^{+}$for all $m=1, \ldots, \mu$.

By Theorem 3.8 and Remarks 3.9 we know

$$
F\left(\tau, c_{\gamma}\right)=\pi\left(C(\gamma, G) \tilde{q}, \frac{V+Z_{V}^{\perp}}{\left|V+Z_{V}^{\perp}\right|}\right) .
$$

Let $v_{p} \in F\left(\tau, c_{\gamma}\right)$. Note that $a_{2}(0)=v$ implies $Z_{0}^{0}=\frac{1}{\tau} Z_{V}^{\perp}$ and $\tau \vartheta_{m}^{0}=\vartheta_{m}\left(Z_{V}^{\perp}\right)$. Also, $X_{2}=0$, since $V \in \operatorname{ker} j\left(Z_{V}^{\perp}\right)=\operatorname{ker} j\left(Z_{0}^{0}\right)$, so that

$$
\xi_{m}^{0}=0
$$

for all $m \in M$.

After substituting these values into (\$) we obtain

$$
\dot{X}^{\prime 0}(r)=\dot{X}_{0}^{0}+\left.\sum_{m} \sin \left(r \vartheta_{m}^{0}\right) \frac{d}{d s}\right|_{0}\left(\frac{1}{\vartheta_{m}^{s}} J_{s} \xi_{m}^{s}\right)+\sum_{m}\left(\cos \left(r \vartheta_{m}^{0}\right)-1\right) \dot{\xi}_{m}^{0}+\left.\sum_{n} r \frac{d}{d s}\right|_{0}\left(J_{s} \xi_{n}^{s}\right) \text {. }
$$

Since this must satisfy condition (6) of Theorem 3.11, after setting $r=\tau$ we obtain

$$
0=\left.\sum_{m} \sin \left(\tau \vartheta_{m}^{0}\right) \frac{d}{d s}\right|_{\left.\right|_{0}}\left(\frac{1}{\vartheta_{m}^{s}} J_{s} \xi_{m}^{s}\right)+\sum_{m}\left(\cos \left(\tau \vartheta_{m}^{0}\right)-1\right) \dot{\xi}_{m}^{0}+\left.\sum_{n} \tau \frac{d}{d s}\right|_{0}\left(J_{s} \xi_{n}^{s}\right) .
$$

Now $\left.\frac{d}{d s}\right|_{0}\left(\frac{1}{\vartheta_{m}^{s}} J_{s} \xi_{m}^{s}\right)=\frac{1}{\vartheta_{m}^{0}} J_{0} \dot{\xi}_{m}^{0}$ since $\xi_{m}^{0}=0$ and we have

$$
0=\sum_{m \in M} \sin \left(\tau \vartheta_{m}^{0}\right) \frac{1}{\vartheta_{m}^{0}} J_{0} \dot{\xi}_{m}^{0}+\sum_{m \in M}\left(\cos \left(\tau \vartheta_{m}^{0}\right)-1\right) \dot{\xi}_{m}^{0}+\left.\sum_{n \in N} \tau \frac{d}{d s}\right|_{0}\left(J_{s} \xi_{n}^{s}\right) .
$$

Note that the right hand side of this equation is an orthogonal decomposition, using linearity of the derivative, the fact that we started with an orthogonal decomposition (before taking derivatives with respect to $s$ ), and the values $J_{0} \xi_{m}^{0}=0$ for all $m$, and $\xi_{m}^{0}=0$ for all $m$. We thus obtain for $m \in M$,

$$
\frac{\sin \left(\tau \vartheta_{m}^{0}\right)}{\vartheta_{m}^{0}} J_{0} \dot{\xi}_{m}^{0}=0, \quad \cos \left(\tau \vartheta_{m}^{0}\right) \dot{\xi}_{m}^{0}=1,
$$

and for $n \in N$,

$$
\left.\frac{d}{d s}\right|_{0}\left(J_{s} \xi_{n}^{s}\right)=0
$$

Next, if there exists $m \in M$ such that $\dot{\xi}_{m}^{0} \neq 0$, then $\sin \left(\tau \vartheta_{m}^{0}\right)=0$ and $\cos \left(\tau \vartheta_{m}^{0}\right)=$ 1 , which implies $\vartheta_{m}\left(Z_{V}^{\perp}\right) \in 2 \pi \mathbb{Z}^{+}$. Since we assumed that $\vartheta_{m}\left(Z_{V}^{\perp}\right) \notin 2 \pi \mathbb{Z}^{+}$for all 
$m=1, \ldots, \mu\left(Z_{V}^{\perp}\right)$, then $\dot{\xi}_{m}^{0}=0$ for all $m \in M$. Since we already know $\xi_{m}^{0}=0$ for all $m$, we may assume $\xi_{m}^{s}=\xi_{m}^{0}=0$ for all $s$ without changing the value of $\dot{a}_{2}(0)$. Substituting these values into $(\boldsymbol{p})$, we obtain

$$
\dot{X}^{0}(\tau)=\tau\left(\dot{X}_{1}^{0}+\sum_{n} \dot{\xi}_{n}^{0}\right)
$$

Since this must satisfy condition (4) of Theorem 3.11, we may assume that

$$
X_{1}^{s}+\sum_{n} \xi_{n}^{s}=V / \tau
$$

for all $s$.

Now by Subsection 3.5,

$$
\dot{Z}^{0}(\tau)=\left.\frac{d}{d s}\right|_{0} \int_{0}^{\tau}\left(Z_{0}^{s}+\frac{1}{2}\left[X^{s}(r), X^{\prime} s(r)\right]\right) d r .
$$

Since everything in sight is analytic in both $s$ and $r$ and bounded with a bounded derivative in $s$ near 0 , and since we are integrating over a finite, closed interval, we can use uniform convergence to bring the derivative $\frac{d}{d s}$ under the integral sign. Using the linearity of the Lie bracket in each component and the product rule, we thus have

$$
\begin{aligned}
\left.\frac{d}{d s}\right|_{0} Z^{s}(\tau) & =\left.\int_{0}^{\tau} \frac{d}{d s}\right|_{0}\left(Z_{0}^{s}+\frac{1}{2}\left[X^{s}(r), X^{\prime s}(r)\right]\right) d t \\
& =\int_{0}^{\tau}\left(\dot{Z}_{0}^{0}+\frac{1}{2}\left[\dot{X}^{0}(r), X^{\prime 0}(r)\right]+\frac{1}{2}\left[X^{0}(r), \dot{X}^{\prime 0}(r)\right]\right) d r \\
& =\tau \dot{Z}_{0}^{0}
\end{aligned}
$$

The fact that $\dot{X}^{0}(r)=0$ and $\dot{X}^{\prime} 0(r)=0$ follows from (\$) and (\$), using the fact that $\xi_{m}^{0}=0, \dot{\xi}_{m}^{0}=0$, and $\left.\frac{d}{d s}\right|_{0}\left(J_{s} \xi_{n}^{s}\right)=0$.

However, this must also satisfy condition (5) of Theorem 3.11, thus $\tau \dot{Z}_{0}^{0}=$ $\left[V, a_{1}(0)\right]$. But also, for $Y \in \mathfrak{v}$,

$$
\begin{aligned}
\left\langle Z_{0}^{s},\left[\frac{1}{\tau} V, Y\right]\right\rangle & =\left\langle Z_{0}^{s},\left[X_{1}^{s}+\sum_{n} \xi_{n}^{s}, Y\right]\right\rangle \\
& =\left\langle J_{s} X_{1}^{s}, Y\right\rangle+\sum_{n}\left\langle J_{s} \xi_{n}^{s}, Y\right\rangle \\
& =\sum_{n}\left\langle J_{s} \xi_{n}^{s}, Y\right\rangle
\end{aligned}
$$

since by the definition of $X_{1}^{s}$, we must have $J_{s} X_{1}^{s}=0$ for all $s$. Taking the derivative of both sides and using the fact that for $n \in N,\left.\frac{d}{d s}\right|_{0} J_{s} \xi_{n}^{s}=0$, we obtain

$$
\dot{Z}_{0}^{0} \perp[V, \mathfrak{g}] .
$$

We conclude that $\dot{Z}_{0}^{0}=0$ and we may assume $Z_{0}^{s}=Z_{0}=Z_{V}^{\perp} / \tau$ for all $s$. This also implies that $\vartheta_{n}\left(Z_{0}^{s}\right)$ is constant, which by definition of the set $N$ implies that $N=\emptyset$. We conclude that $X_{1}^{s}=V / \tau$ for all $s$ and $X_{2}^{s}=0$ for all $s$. We also conclude that $\left[V, \dot{a}_{1}(0)\right]=0$ and $\dot{a}_{1}(0)$ may be represented by a curve in $C(\gamma, G)=\{\exp (X)$ : $[X, V]=0, X \in \mathfrak{g}\}$. 
That is,

$$
\left(\dot{a}_{1}(0), \dot{a}_{2}(0)\right)_{v_{\tilde{p}}} \in T_{v_{\tilde{p}}} \tilde{F}(\tau, \gamma),
$$

where $\tilde{F}(\tau, \gamma)$ is as in $(\mathcal{Q})$.

We have shown that if $\vartheta_{m}\left(Z_{V}^{\perp}\right) \notin 2 \pi \mathbb{Z}^{+}$for all $m=1, \ldots, \mu\left(Z_{V}^{\perp}\right)$, then $\tau=$ $\left|V+Z_{V}^{\perp}\right|$ satisfies the Clean Intersection Hypothesis.

Case II: Let $v_{\tilde{p}} \in \tilde{F}(\tau, \gamma)$ with $\tau=\sqrt{|V|^{2}+\left|Z_{V}^{\perp}\right|^{2} /\left|K_{V}^{\perp}\left(\bar{X}_{2}+\bar{Z}_{0}\right)\right|^{2}}$ with $\bar{X}_{2}+\bar{Z}_{0}$ satisfying (i)-(v) of Theorem 3.8. We exclude the possibility that $\tau=\left|V+Z_{V}^{\perp}\right|$ from this case.

Recall that we assume that $\alpha(s)=\left(\tilde{p} \exp \left(a_{1}(s)\right), a_{2}(s)\right) \in \tilde{\mathfrak{X}}$ with $a_{1}(0)=0$ and $a_{2}(0)=v=X_{1}+X_{2}+Z_{0}$ and that $\tilde{\Phi}_{\tau *_{\tilde{\tilde{n}}}}(\dot{\alpha}(0))=L_{\gamma *} \dot{\alpha}(0)$. With notation as above, $a_{2}(s)=X_{1}^{s}+X_{2}^{s}+Z_{0}^{s}$, where $Z_{0}^{s} \in \mathfrak{z}, X_{1}^{s}+X_{2}^{s} \perp \mathfrak{z}, X_{1}^{s} \in \operatorname{ker} j\left(Z_{0}^{s}\right)$ and $X_{2}^{s} \perp \operatorname{ker} j\left(Z_{0}^{s}\right)$. In addition, $X_{2}^{s}=\sum_{n \in N} \xi_{n}^{s}+\sum_{m \in M} \xi_{m}^{s}$, where $\xi_{m}^{s} \in W_{m}\left(Z_{0}^{s}\right)$ and $\xi_{n}^{s} \in W_{n}\left(Z_{0}^{s}\right)$ as above. The geodesic with initial velocity $\alpha(s)$ can be written as $\tilde{p} \exp \left(a_{1}(s)\right) \exp \left(X^{s}(t)+Z^{s}(t)\right)$, with $a_{1}(0)=0$, where $X^{s}(t)$ and $X^{\prime s}(t)$ satisfy $(\boldsymbol{\beta})$ and $(\mathbf{\$})$, respectively.

We use the fact that $\dot{\alpha}(0)$ satisfies conditions (1) through (6) of Theorem 3.11 to show that $\dot{\alpha}(0)$ is tangent to $\tilde{F}(\tau, \gamma)$ and hence $\pi_{*}(\dot{\alpha}(0))$ is tangent to $F\left(\tau, c_{\gamma}\right)$.

Since condition (1) of Theorem 3.11 must hold, and since by Theorem 3.8 $J\left(Z_{0}^{0}\right) V=0$, and since $\xi_{m}^{0} \perp V$ for all $m=1, \ldots, \mu$, we must have the condition: if $\xi_{m}^{0} \neq 0$, then $\tau \vartheta_{m}^{0} \in 2 \pi \mathbb{Z}^{+}$. However, if $\xi_{m}^{0}=0$ but $\dot{\xi}_{m}^{0} \neq 0$, we cannot yet make this conclusion. We thus divide the set $M$ into two disjoint subsets:

$$
\begin{aligned}
& M^{\prime}=\left\{m \in M: \xi_{m}^{0} \neq 0\right\}, \\
& M^{\prime \prime}=\left\{m \in M: \xi_{m}^{0}=0\right\} .
\end{aligned}
$$

We thus have $\tau \vartheta_{m}^{0} \in 2 \pi \mathbb{Z}^{+}$for $m \in M^{\prime}$. Substituting this into ( $\left.\mathbf{(}\right)$ we obtain

$$
\begin{aligned}
\dot{X}^{\prime 0}(\tau) & =\left(\dot{X}_{1}^{0}+\sum_{n} \dot{\xi}_{n}^{0}\right)+\sum_{m \in M^{\prime}} \tau \dot{\vartheta}_{m}^{0} \frac{1}{\vartheta_{m}^{0}} J_{0} \xi_{m}^{0}+\sum_{m \in M^{\prime}} \dot{\xi}_{m}^{0} \\
& +\left.\sum_{m \in M^{\prime \prime}} \sin \left(\tau \vartheta_{m}^{0}\right) \frac{d}{d s}\right|_{\left.\right|_{0}}\left(\frac{1}{\vartheta_{m}^{s}} J_{s} \xi_{m}^{s}\right)+\sum_{m \in M^{\prime \prime}} \cos \left(\tau \vartheta_{m}^{0}\right) \dot{\xi}_{m}^{0}+\left.\sum_{n} \tau \frac{d}{d s}\right|_{\left.\right|_{0}}\left(J_{s} \xi_{n}^{s}\right) .
\end{aligned}
$$

Since this must satisfy condition (6) of Theorem 3.11 , we obtain

$$
\begin{aligned}
0 & =\sum_{m \in M^{\prime}} \tau \dot{\vartheta}_{m}^{0} \frac{1}{\vartheta_{m}^{0}} J_{0} \xi_{m}^{0}+\left.\sum_{m \in M^{\prime \prime}} \sin \left(\tau \vartheta_{m}^{0}\right) \frac{d}{d s}\right|_{\left.\right|_{0}}\left(\frac{1}{\vartheta_{m}^{s}} J_{s} \xi_{m}^{s}\right) \\
& +\sum_{m \in M^{\prime \prime}}\left(\cos \left(\tau \vartheta_{m}^{0}\right)-1\right) \dot{\xi}_{m}^{0}+\left.\sum_{n} \tau \frac{d}{d s}\right|_{\left.\right|_{0}}\left(J_{s} \xi_{n}^{s}\right) .
\end{aligned}
$$

As in Case I, this is an orthogonal decomposition. We first conclude that

$$
\left.\frac{d}{d s}\right|_{0}\left(J_{s} \xi_{n}^{s}\right)=0
$$

for all $n \in N$. Also, if $X_{2}^{0}=0$, then $\tau=\left|V+Z_{V}^{\perp}\right|$, which was included in Case I and excluded from Case II, so $X_{2}^{0} \neq 0$. Since $X_{2}^{0} \perp \operatorname{ker}\left(J_{0}\right), J_{0} X_{2}^{0} \neq 0$, and since $J_{0} \xi_{m}^{0} \perp J_{0} \xi_{m^{\prime}}^{0}$ when $m \neq m^{\prime}$, we conclude that

$$
\dot{\vartheta}_{m}^{0}=0
$$


for all $m \in M^{\prime}$. Finally, if $\xi_{m}^{0}=0$ and $\dot{\xi}_{m}^{0} \neq 0$, then $\tau \vartheta_{m}^{0} \in 2 \pi \mathbb{Z}^{+}$; that is, for all $m \in M$, if $\xi_{m}^{0} \neq 0$ or $\dot{\xi}_{m}^{0} \neq 0$, then $\tau \vartheta_{m}^{0} \in 2 \pi \mathbb{Z}^{+}$. If $\xi_{m}^{0}=0$ and $\dot{\xi}_{m}^{0}=0$, then we may conclude that $\xi_{m}^{s} \equiv 0$ and in particular $\left.\frac{d}{d s}\right|_{0}\left(\frac{1}{\vartheta_{m}^{s}} J_{s} \xi_{m}^{s}\right)=0$.

Substituting these values into (\$) we obtain

$$
\dot{X}^{0}(\tau)=\tau\left(\dot{X}_{1}^{0}+\sum_{n} \dot{\xi}_{n}^{0}\right)
$$

Since $\dot{X}^{0}(\tau)$ must satisfy condition (4) of Theorem 3.11, we conclude $\dot{X}_{1}^{0}+\sum_{n} \dot{\xi}_{n}^{0}=$ 0 . This implies that we may assume that $X_{1}^{s}+\sum_{n} \xi_{n}^{s}=V / \tau$ for all $s$.

We now claim that $\dot{Z}_{0}^{0} \perp[V, \mathfrak{g}]$. To see this, note that $Z_{0}^{0} \perp[V, \mathfrak{g}]$ by Theorem 3.7. Thus for $Y \in \mathfrak{g}$,

$$
\begin{aligned}
\left\langle\dot{Z}_{0}^{0},\left[\frac{1}{\tau} V, Y\right]\right\rangle & =\lim _{s \rightarrow 0}\left\langle\frac{1}{s}\left(Z_{0}^{s}-Z_{0}^{0}\right),\left[\frac{1}{\tau} V, Y\right]\right\rangle \\
& =\lim _{s \rightarrow 0}\left\langle\frac{1}{s} Z_{0}^{s},\left[X_{1}^{s}+\sum_{n} \xi_{n}^{s}, Y\right]\right\rangle \\
& =\lim _{s \rightarrow 0}\left\langle\frac{1}{s}\left(J_{s} X_{1}^{s}+\sum_{n} J_{s} \xi_{n}^{s}\right), Y\right\rangle \\
& =\lim _{s \rightarrow 0}\left\langle\sum_{n} \frac{1}{s} J_{s} \xi_{n}^{s}, Y\right\rangle \\
& =\sum_{n}\left\langle\left.\frac{d}{d s}\right|_{0}\left(J_{s} \xi_{n}^{s}\right), Y\right\rangle=0 .
\end{aligned}
$$

Since $Z_{0}^{0} \perp[V, \mathfrak{g}]$ and $\dot{Z}_{0}^{0} \perp[V, \mathfrak{g}]$, we may now replace the central component $Z_{0}^{s}$ of $a_{2}(s)$ with a curve that is contained in the orthogonal complement of $[V, \mathfrak{g}]$ without changing the value of $Z_{0}$ or $\dot{Z}_{0}^{0}$. That is, we now assume that $Z_{0}^{s} \perp[V, \mathfrak{g}]$ for all $s$. This implies $J_{s} V=0$ for all $s$, and since by the definition of $X_{1}^{s}, J_{s} X_{1}^{s}=0$ for all $s$, we conclude that $J_{s} \xi_{n}^{s}=0$ for all $s$. However, for $s \neq 0, \vartheta_{n}^{s} \neq 0$ by the definition of the set $N$. We conclude that $\xi_{n}^{s} \equiv 0$ for all $n \in N$, that is, $\xi_{n}^{s} \equiv 0$ and $\dot{\xi}_{n}^{0}=0$ for all $n \in N$ and $X_{2}^{s}=\sum_{m \in M} \xi_{m}^{s}$ for all $s$.

We have shown that we may assume that $X_{1}^{s} \equiv V / \tau$ for all $s$.

Since $\left|X_{1}^{s}\right|$ is constant and $\left|a_{2}(s)\right| \equiv 1$, we must have $\left|X_{2}^{s}+Z_{0}^{s}\right|$ constant. In particular,

$$
\left|X_{2}^{s}+Z_{0}^{s}\right|=\frac{\sqrt{\tau^{2}-|V|^{2}}}{\tau}
$$

We define

$$
\begin{aligned}
& \bar{X}_{2}^{s}=\frac{\tau}{\sqrt{\tau^{2}-|V|^{2}}} X_{2}^{s}, \\
& \bar{Z}_{0}^{s}=\frac{\tau}{\sqrt{\tau^{2}-|V|^{2}}} Z_{0}^{s},
\end{aligned}
$$

i.e., $\bar{X}_{2}^{s}+\bar{Z}_{0}^{s}$ is a unit vector in the same direction as $X_{2}^{s}+Z_{0}^{s}$. Note that $\bar{X}_{2}^{0}=\bar{X}_{2}$ and $\bar{Z}_{0}^{0}=\bar{Z}_{0}$.

We have shown that we may assume that

$$
X_{1}^{s}+X_{2}^{s}+Z_{0}^{s}=\frac{1}{\tau}\left(V+\sqrt{\tau^{2}-|V|^{2}}\left(\bar{X}_{2}^{s}+\bar{Z}_{0}^{s}\right)\right)
$$


for all $s$, that $\left|\bar{X}_{2}^{s}+\bar{Z}_{0}^{s}\right|=1$ for all $s$, and that $V \in \operatorname{ker} J_{s}$ and $\bar{X}_{2}^{s} \perp \operatorname{ker} J_{s}$ for all $s$; i.e., we have shown that conditions (i) and (ii) of Theorem 3.8 are satisfied. It remains to show that conditions (iii), (iv), and (v) are likewise satisfied.

Substituting $\dot{X}_{1}^{0}=0, \dot{\vartheta}_{m}^{0}=0$ for all $m \in M$, and $\xi_{n}^{s} \equiv 0$ for all $n \in N$ into and $(\mathbf{\uparrow})$, we obtain

$$
\dot{X}^{0}(r)=\left.\sum_{m \in M} \frac{1-\cos \left(r \vartheta_{m}^{0}\right)}{\vartheta_{m}^{0}} \frac{d}{d s}\right|_{\left.\right|_{0}}\left(\frac{1}{\vartheta_{m}^{s}} J_{s} \xi_{m}^{s}\right)+\sum_{m \in M} \frac{\sin \left(r \vartheta_{m}^{0}\right)}{\vartheta_{m}^{0}} \dot{\xi}_{m}^{0}
$$

and

$$
\dot{X}^{\prime 0}(r)=\left.\sum_{m \in M} \sin \left(r \vartheta_{m}^{0}\right) \frac{d}{d s}\right|_{0}\left(\frac{1}{\vartheta_{m}^{s}} J_{s} \xi_{m}^{s}\right)+\sum_{m \in M} \cos \left(r \vartheta_{m}^{0}\right) \dot{\xi}_{m}^{0}
$$

We also easily show that

$$
X^{0}(r)=\frac{r}{\tau} V+\sum_{m \in M} \frac{1-\cos \left(r \vartheta_{m}^{0}\right)}{\vartheta_{m}^{0}} \frac{1}{\vartheta_{m}^{0}} J_{0} \xi_{m}^{0}+\sum_{m \in M} \frac{\sin \left(r \vartheta_{m}^{0}\right)}{\vartheta_{m}^{0}} \xi_{m}^{0}
$$

and

$$
X^{\prime 0}(r)=\frac{1}{\tau} V+\sum_{m \in M} \sin \left(r \vartheta_{m}^{0}\right) \frac{1}{\vartheta_{m}^{0}} J_{0} \xi_{m}^{0}+\sum_{m \in M} \cos \left(r \vartheta_{m}^{0}\right) \xi_{m}^{0}
$$

As in Case I,

$$
\dot{Z}^{0}(\tau)=\left.\frac{d}{d s}\right|_{0} \int_{0}^{\tau}\left(Z_{0}^{s}+\frac{1}{2}\left[X^{s}(r), X^{\prime} s(r)\right]\right) d r .
$$

Since everything in sight is analytic in both $s$ and $r$ and a bounded with a bounded derivative in $s$ near 0 , and since we are integrating over a finite, closed interval, we can use uniform convergence to bring the derivative $\frac{d}{d s}$ under the integral sign. Using the linearity of the Lie bracket in each component and the product rule, we thus have

$$
\begin{aligned}
\left.\frac{d}{d s}\right|_{0} Z^{s}(\tau) & =\left.\int_{0}^{\tau} \frac{d}{d s}\right|_{0}\left(Z_{0}^{s}+\frac{1}{2}\left[X^{s}(r), X^{\prime s}(r)\right]\right) d r \\
& =\int_{0}^{\tau}\left(\dot{Z}_{0}^{0}+\frac{1}{2}\left[\dot{X}^{0}(r), X^{\prime 0}(r)\right]+\frac{1}{2}\left[X^{0}(r), \dot{X}^{\prime 0}(r)\right]\right) d r .
\end{aligned}
$$


After substituting in the above values of $X^{0}(r), X^{\prime 0}(r), \dot{X}^{0}(r)$ and $\dot{X}^{\prime 0}(r)$, we obtain

$$
\begin{aligned}
& 2\left(\dot{Z}^{0}(\tau)-\tau \dot{Z}_{0}^{0}\right)=\frac{1}{\tau} \sum_{m \in M} \int_{0}^{\tau}\left(r \cos \left(r \vartheta_{m}^{0}\right)-\frac{\sin \left(r \vartheta_{m}^{0}\right)}{\vartheta_{m}^{0}}\right) d r\left[V, \dot{\xi}_{m}^{0}\right] \\
& +\frac{1}{\tau} \sum_{m \in M} \int_{0}^{\tau}\left(r \sin \left(r \vartheta_{m}^{0}\right)-\frac{1-\cos \left(r \vartheta_{m}^{0}\right)}{\vartheta_{m}^{0}}\right) d r\left[V,\left.\frac{d}{d s}\right|_{0}\left(\frac{1}{\vartheta_{m}^{s}} J_{s} \xi_{m}^{s}\right)\right] \\
& +\sum_{m, m^{\prime} \in M} \int_{0}^{\tau}\left(\frac{\sin \left(r \vartheta_{m}^{0}\right) \cos \left(r \vartheta_{m^{\prime}}^{0}\right)}{\vartheta_{m}^{0}}-\frac{\cos \left(r \vartheta_{m}^{0}\right) \sin \left(r \vartheta_{m^{\prime}}^{0}\right)}{\vartheta_{m^{\prime}}^{0}}\right) d r\left[\xi_{m}^{0}, \dot{\xi}_{m^{\prime}}^{0}\right] \\
& +\sum_{m, m^{\prime} \in M} \int_{0}^{\tau}\left(\frac{\left(1-\cos \left(r \vartheta_{m}^{0}\right)\right) \sin \left(r \vartheta_{m^{\prime}}^{0}\right)}{\vartheta_{m}^{0}}-\frac{\sin \left(r \vartheta_{m}^{0}\right)\left(1-\cos \left(r \vartheta_{m^{\prime}}^{0}\right)\right)}{\vartheta_{m^{\prime}}^{0}}\right) d r \\
& +\sum_{m, m^{\prime} \in M} \int_{0}^{\tau}\left(\frac{\sin \left(r \vartheta_{m}^{0}\right) \sin \left(r \vartheta_{m^{\prime}}^{0}\right)}{\vartheta_{m}^{0}}-\frac{\cos \left(r \vartheta_{m}^{0}\right)\left(1-\cos \left(r \vartheta_{m^{\prime}}^{0}\right)\right)}{\vartheta_{m^{\prime}}^{0}} J_{0} \xi_{m}^{0},\left.\frac{d}{d s}\right|_{\left.\right|_{0} ^{\prime}}\left(\frac{1}{\vartheta_{m^{\prime}}^{s}} J_{s} \xi_{m^{\prime}}^{s}\right)\right] \\
& \left.\cdot \frac{d}{d s}\right|_{\left.\right|_{0}}\left(\left[\xi_{m}^{s}, \frac{1}{\vartheta_{m^{\prime}}^{s}} J_{s} \xi_{m^{\prime}}^{s}\right]\right) .
\end{aligned}
$$

Note that for all $m, m^{\prime} \in M$, the integrals in front of the terms $\left[V, \dot{\xi}_{m}^{0}\right],\left[\xi_{m}^{0}, \dot{\xi}_{m^{\prime}}^{0}\right]$ and $\left[\frac{1}{\vartheta_{m}^{0}} J_{0} \xi_{m}^{0},\left.\frac{d}{d s}\right|_{0}\left(\frac{1}{\vartheta_{m^{\prime}}^{s}} J_{s} \xi_{m^{\prime}}^{s}\right)\right]$ are always zero. For all $m \in M$, the integral in front of the term $\left[V,\left.\frac{d}{d s}\right|_{0}\left(\frac{1}{\vartheta_{m}^{s}} J_{s} \xi_{m}^{s}\right)\right]$ is equal to $-2 \tau / \vartheta_{m}^{0}$. Finally, for all $m, m^{\prime} \in M$, if $\vartheta_{m}^{0} \neq \vartheta_{m^{\prime}}^{0}$, then the integral in front of the term $\left.\frac{d}{d s}\right|_{0}\left(\left[\xi_{m}^{s}, \frac{1}{\vartheta_{m^{\prime}}^{s}} J_{s} \xi_{m^{\prime}}^{s}\right]\right)$ is equal to 0 . If $\vartheta_{m}^{0}=\vartheta_{m^{\prime}}^{0}$, the integral in front of the term is $\tau / \vartheta_{m}^{0}$. At this point we must consider refined invariant subspaces. To do so, we define the set $R$ as follows:

$$
R:=\left\{\left(m, m^{\prime}\right): m, m^{\prime} \in M, m<m^{\prime}, \vartheta_{m}^{0}=\vartheta_{m^{\prime}}^{0}\right\} .
$$

We thus have

$$
\begin{aligned}
\dot{Z}^{0}(\tau) & =\tau \dot{Z}_{0}^{0}-\sum_{m \in M} \frac{1}{\vartheta_{m}^{0}}\left[V,\left.\frac{d}{d s}\right|_{\left.\right|_{0}}\left(\frac{1}{\vartheta_{m}^{s}} J_{s} \xi_{m}^{s}\right)\right]+\left.\sum_{m \in M} \frac{\tau}{2 \vartheta_{m}^{0}} \frac{d}{d s}\right|_{\left.\right|_{0}}\left(\left[\xi_{m}^{s}, \frac{1}{\vartheta_{m}^{s}} J_{s} \xi_{m}^{s}\right]\right) \\
& +\left.\sum_{\left(m, m^{\prime}\right) \in R} \frac{\tau}{2 \vartheta_{m}^{0}} \frac{d}{d s}\right|_{\left.\right|_{0}}\left(\left[\xi_{m}^{s}, \frac{1}{\vartheta_{m^{\prime}}^{s}} J_{s} \xi_{m^{\prime}}^{s}\right]+\left[\xi_{m^{\prime}}^{s}, \frac{1}{\vartheta_{m}^{s}} J_{s} \xi_{m}^{s}\right]\right) .
\end{aligned}
$$

Since either $J_{0} \xi_{m}^{0}=0$ (if $m \in M^{\prime \prime}$ ) or $\dot{\vartheta}_{m}^{0}=0$ (if $m \in M^{\prime}$ ), we must have

$$
\left.\frac{d}{d s}\right|_{0} \frac{1}{\left(\vartheta_{m}^{s}\right)^{2}} J_{s} \xi_{m}^{s}=\left.\frac{1}{\left(\vartheta_{m}^{0}\right)^{2}} \frac{d}{d s}\right|_{0}\left(J_{s} \xi_{m}^{s}\right) .
$$

Thus by part (2) of Notation 3.2,

$$
\left.\frac{-1}{\vartheta_{m}^{0}} \frac{d}{d s}\right|_{0}\left(J_{s} \xi_{m}^{s}\right)=\left.\frac{d}{d s}\right|_{\left.\right|_{0}}\left(J_{s}^{-1} \xi_{m}^{s}\right)
$$


We now have

$$
\begin{aligned}
\dot{Z}^{0}(\tau) & =\tau \dot{Z}_{0}^{0}+\left.\frac{d}{d s}\right|_{0}\left[V, J_{s}^{-1} X_{2}^{s}\right]+\left.\sum_{m \in M} \frac{\tau}{2} \frac{d}{d s}\right|_{0}\left(\left[J_{s}^{-1} \xi_{m}^{s}, \xi_{m}^{s}\right]\right) \\
& +\left.\sum_{\left(m, m^{\prime}\right) \in R} \frac{\tau}{2 \vartheta_{m}^{0}} \frac{d}{d s}\right|_{0}\left(\left[\xi_{m}^{s}, J_{s} \xi_{m^{\prime}}^{s}\right]+\left[\xi_{m^{\prime}}^{s}, J_{s} \xi_{m}^{s}\right]\right) .
\end{aligned}
$$

We claim that for $\left(m, m^{\prime}\right) \in R,\left.\frac{d}{d s}\right|_{0}\left(\left[\xi_{m}^{s}, J_{s} \xi_{m^{\prime}}^{s}\right]+\left[\xi_{m^{\prime}}^{s}, J_{s} \xi_{m}^{s}\right]\right)=0$. To see this, note that by Proposition 3.14, $\left[\xi_{m}^{0}, J_{0} \xi_{m^{\prime}}^{0}\right]+\left[\xi_{m^{\prime}}^{0}, J_{0} \xi_{m}^{0}\right]=0$. By $[\mathrm{LP}$ (see also [GtM1, p. 205]), for all $\hat{Z} \in \mathfrak{z}$,

$$
\begin{aligned}
\left\langle\hat{Z},\left[\xi_{m}^{s}, J_{s} \xi_{m^{\prime}}^{s}\right]+\left[\xi_{m^{\prime}}^{s}, J_{s} \xi_{m}^{s}\right]\right\rangle & =-\left\langle\left(j(\hat{Z}) J_{s}+J_{s} j(\hat{Z})\right) \xi_{m}^{s}, \xi_{m^{\prime}}^{s}\right\rangle \\
& =\left|\xi_{m}^{s}\right|\left|\xi_{m^{\prime}}^{s}\right|\left(\left(\vartheta_{m^{\prime}}^{s}\right)^{2}-\left(\vartheta_{m}^{s}\right)^{2}\right)\left\langle\frac{\partial \hat{\xi}_{m}^{s}}{\partial z}, \hat{\xi}_{m^{\prime}}^{s}\right\rangle,
\end{aligned}
$$

where

$$
\hat{\xi}_{m}^{s}=\frac{1}{\left|\xi_{m}^{s}\right|} \xi_{m}^{s}
$$

and $\partial \hat{\xi}_{m}^{s} / \partial z$ is the derivative of $\hat{\xi}_{m}^{s}$ in the direction $\hat{Z}$ evaluated at $Z_{0}^{s}$. To be precise, we consider an analytic unit eigenvector curve $\hat{\xi}(Z)$ defined for $Z=Z_{0}^{s}+t \hat{Z}$ in a neighborhood of $t$, that is equal to $\hat{\xi}_{m}^{s}$ at $Z=Z_{0}^{s}$. We then take the derivative of $\hat{\xi}\left(Z_{0}^{s}+t \hat{Z}\right)$ with respect to $t$ at zero. While this may not be well defined, it is well defined up to a choice of eigenvector curve $\hat{\xi}(Z)$. As the existence rather than the value of this curve is what we need, we may proceed. See $\mathrm{Kt}$ and $\mathrm{A}]$ for details about eigenvalue curves and eigenvector curves. See [GtM1, page 205] for the use of these concepts in this context.

Thus

$$
\begin{aligned}
\left\langle\hat{Z},\left.\frac{d}{d s}\right|_{0}\left(\left[\xi_{m}^{s}, J_{s} \xi_{m^{\prime}}^{s}\right]+\left[\xi_{m^{\prime}}^{s}, J_{s} \xi_{m}^{s}\right]\right)\right\rangle & =\left.\frac{d}{d s}\right|_{0}\left\langle\hat{Z},\left[\xi_{m}^{s}, J_{s} \xi_{m^{\prime}}^{s}\right]+\left[\xi_{m^{\prime}}^{s}, J_{s} \xi_{m}^{s}\right]\right\rangle \\
& =\left.\frac{d}{d s}\right|_{0}\left(\left|\xi_{m}^{s} \| \xi_{m^{\prime}}^{s}\right|\left(\left(\vartheta_{m^{\prime}}^{s}\right)^{2}-\left(\vartheta_{m}^{s}\right)^{2}\right)\left\langle\frac{\partial \hat{\xi}_{m}^{s}}{\partial z}, \hat{\xi}_{m^{\prime}}^{s}\right\rangle\right) .
\end{aligned}
$$

Since $\vartheta_{m}^{0}=\vartheta_{m^{\prime}}^{0}$, the derivatives of $\left|\xi_{m}^{s}\right|,\left|\xi_{m^{\prime}}^{s}\right|$ and $\left\langle\frac{\partial \hat{\xi}_{m}^{s}}{\partial z}, \hat{\xi}_{m^{\prime}}^{s}\right\rangle$ do not enter into the computation. We only need the fact that these derivatives exist. Note that for $m, m^{\prime} \in M^{\prime}$, since $\dot{\vartheta}_{m}^{0}=0$, and $\dot{\vartheta}_{m^{\prime}}^{0}=0$,

$$
\left.\frac{d}{d s}\right|_{0}\left(\left(\vartheta_{m^{\prime}}^{s}\right)^{2}-\left(\vartheta_{m}^{s}\right)^{2}\right)=0
$$

If either $m \in M^{\prime \prime}$, or $m^{\prime} \in M^{\prime \prime}$, we still obtain the desired result since either $\left|\xi_{m}^{0}\right|=0$ or $\left|\xi_{m^{\prime}}^{0}\right|=0$.

We conclude that for $\left(m, m^{\prime}\right) \in R$,

$$
\left.\frac{d}{d s}\right|_{0}\left(\left[\xi_{m}^{s}, J_{s} \xi_{m^{\prime}}^{s}\right]+\left[\xi_{m^{\prime}}^{s}, J_{s} \xi_{m}^{s}\right]\right)=0 .
$$

Since $\left[\xi_{m}^{0}, J_{0} \xi_{m^{\prime}}^{0}\right]+\left[\xi_{m^{\prime}}^{0}, J_{0} \xi_{m}^{0}\right]$ and $\left.\frac{d}{d s}\right|_{0}\left(\left[\xi_{m}^{s}, J_{s} \xi_{m^{\prime}}^{s}\right]+\left[\xi_{m^{\prime}}^{s}, J_{s} \xi_{m}^{s}\right]\right)$ depend only on $Z_{0}^{0}, \dot{Z}_{0}^{0}, X_{2}^{0}$, and $\dot{X}_{2}^{0}$, we may replace the curve $X_{2}^{s}+Z_{0}^{s}$ by one such that $\left[\xi_{m}^{s}, J_{s} \xi_{m^{\prime}}^{s}\right]+$ $\left[\xi_{m^{\prime}}^{s}, J_{s} \xi_{m}^{s}\right]=0$ for all $s$ without changing the value of $\dot{a}_{2}(0)$. 
We have also shown that

$$
\dot{Z}^{0}(\tau)=\left.\tau \frac{d}{d s}\right|_{0}\left(K\left(X_{2}^{s}+Z_{0}^{s}\right)+\left[V, J_{s}^{-1} X_{2}^{s}\right]\right) .
$$

Since condition (6) of Theorem 3.11 must be satisfied, $\dot{Z}^{0}(\tau) \subset[V, \mathfrak{g}]$, so that we must have $\left.\frac{d}{d s}\right|_{0} K_{V}^{\perp}\left(X_{2}^{s}+Z_{0}^{s}\right)=0$. Since $\left|X_{2}^{s}+Z_{0}^{s}\right|$ is constant, the values $K_{V}^{\perp}\left(\bar{X}_{2}^{0}+\bar{Z}_{0}^{0}\right)=\frac{1}{\sqrt{\tau^{2}-|V|^{2}}} Z_{V}^{\perp}$ and $\left.\frac{d}{d s}\right|_{0} K_{V}^{\perp}\left(\bar{X}_{2}^{s}+\bar{Z}_{0}^{s}\right)$ depend only on $Z_{0}^{0}, \dot{Z}_{0}^{0}, X_{2}^{0}$ and $\dot{X}_{2}^{0}$. As above we may assume that

$$
K_{V}^{\perp}\left(\bar{X}_{2}^{s}+\bar{Z}_{0}^{s}\right) \equiv \frac{1}{\sqrt{\tau^{2}-|V|^{2}}} Z_{V}^{\perp}
$$

without changing the value of $\dot{a}_{2}(0)$, and condition (iii) of ( is satisfied for all $s$.

Now by [LP], $\dot{\vartheta}_{m}^{0}=\nabla \vartheta_{m}\left(Z_{0}\right) \cdot \dot{Z}_{0}^{0}$, where $\nabla \vartheta_{m}(Z)$ is the gradient of the curve $\vartheta_{m}(Z)$ at $Z \in \mathfrak{z}$. Since $\dot{\vartheta}_{m}^{0}$ and $\vartheta_{m}^{0}$ depend only on $Z_{0}^{0}$ and $\dot{Z}_{0}^{0}$, and since $\dot{\vartheta}_{m}^{0}=0$ for all $m \in M$, as above, we may replace $Z_{0}^{s}$ by a curve such that

$$
\vartheta_{m}\left(\bar{Z}_{0}^{s}\right) \in \frac{2 \pi}{\sqrt{\tau^{2}-|V|^{2}}} \mathbb{Z}^{+}
$$

for all $s$, and condition (iv) of (

Now $\dot{Z}^{0}(\tau)=\left.\frac{d}{d s}\right|_{0}\left(\left[V, j\left(Z_{0}^{s}\right)^{-1} X_{2}^{s}\right]+\tau K_{V}\left(X_{2}^{s}+Z_{0}^{s}\right)\right)$ but $\dot{Z}^{0}(\tau)=\left[V, \dot{a}_{1}(0)\right]$ by part (5) of Theorem 3.11. So,

$$
\left[V, \dot{a}_{1}(0)\right]=\left.\frac{d}{d s}\right|_{0}\left(\left[V, j\left(Z_{0}^{s}\right)^{-1} X_{2}^{s}\right]+\tau K_{V}\left(X_{2}^{s}+Z_{0}^{s}\right)\right) .
$$

Since the values $K_{V}\left(X_{2}^{0}+Z_{0}^{0}\right)+\left[V, J_{0}^{-1} X_{2}^{0}\right]$ and $\left.\frac{d}{d s}\right|_{0}\left(K_{V}\left(X_{2}^{s}+Z_{0}^{s}\right)+\left[V, J_{s}^{-1} X_{2}^{s}\right]\right)$ depend only on the values $X_{2}^{0}, Z_{2}^{0}, \dot{X}_{2}^{0}$ and $\dot{Z}_{0}^{0}$, we may replace the curve $X_{2}^{s}+Z_{0}^{s}$ with one such that $K_{V}\left(X_{2}^{s}+Z_{0}^{s}\right)+\left[V, J_{s}^{-1} X_{2}^{s}\right]$ is constant for all $s$. After normalizing to $\bar{X}_{2}^{s}+\bar{Z}_{0}^{s}$ and using the fact that $\left|X_{2}^{s}+Z_{0}^{s}\right|$ is constant, we see that we may assume that condition (v) of ( $)$ is satisfied for all $s$.

In conclusion,

$$
\left(\dot{a}_{1}(0), \dot{a}_{2}(0)\right)_{v_{\tilde{p}}}
$$

is tangent to a curve in $\tilde{F}(\tau, \gamma)$, as desired. Thus, there cannot exist "unclean" lengths coming from Case II.

\section{REFERENCES}

[A] P. Anselone, Collectively Compact Operator Approximation Theory and Applications to Integral Equations, Prentice Hall, 1971. Mr 0443383 (56:1753)

[BGM] M. Berger, P. Gauduchon, and E. Mazet, Le spectre d'une variété Riemannienne, Lecture Notes in Mathematics, vol. 194, Springer-Verlag, 1971. MR0282313 (43:8025)

[Bs] A. L. Besse, Manifolds all of whose Geodesics are Closed, Springer-Verlag, 1978. MR0496885 (80c:53044)

[Bu] P. Buser, Geometry and Spectra of Compact Riemann Surfaces, Birkhäuser, 1992. MR 1183224 (93g:58149)

[Ch] I. Chavel, Eigenvalues in Riemannian Geometry, Academic Press, 1984. MR0768584 (86g:58140)

[CdV] Y. Colin de Verdière, Spectre du laplacien et longueurs des géodésiques périodiques, I-II, Compositio Math 27 (1973), 83-106, 159-184. MR0348798 (50:1293)

[DG1] D. DeTurck and C. S. Gordon, Isospectral Deformations I: Riemannian structures on twostep nilspaces, Comm. Pure Appl. Math. 40 (1987), 367-387. MR.0882070 (88m:58186) 
[DG2] - Isospectral Deformations II: trace formulas, metrics, and potentials, Comm. Pure Appl. Math. 42 (1989), 1067-1095. MR1029118 (91e:58197)

[DGu] J. J. Duistermaat and V. W. Guillemin, The spectrum of poitive elliptic operators and periodic bicharacteristics, Invent. Math. 29 (1977), 39-79. MR0405514 (53:9307)

[E1] P. Eberlein, Geometry of two-step nilpotent groups with a left invariant metric, Ann. Sci. de École Norm. Sup. 27 (1994), 611-660. MR.1296558 (95m:53059)

[E2] Geometry of two-step nilpotent groups with a left invariant metric II, Trans. AMS 343 (1994), 805-828. MR1250818(95b:53061)

[G1] C. S. Gordon, The Laplace spectra versus the length spectra of Riemannian manifolds, Contemporary Mathematics 51 (1986), 63-79. MR0848934 (87i:58170)

[G2] , Riemannian manifolds isospectral on functions but not on 1-forms, J. Diff. Geom. 24 (1986), 79-96. MR0857377 (87m:58174)

[G3] - Isospectral closed Riemannian manifolds which are not locally isometric, J. Diff. Geom. 37 (1993), 639-649. MR1217163 (94b:58098)

[G4] I Isospectral deformations of metrics on spheres, Invent. Math. 145 (2001), 317331. MR.1872549 (2003d:58052)

[G5] _ Isospectral manifolds with different local geometry, J. Korean Math. Soc. 38 (2001), no. 5, 955-970. MR1849334 (2002f:58058)

[GW1] C. S. Gordon and E. N. Wilson, Isospectral deformations of compact solvmanifolds, J. Differential Geometry 19 (1984), 241-256. MR0739790 (85j:58143)

[GW2] _ The spectrum of the Laplacian on Riemannian Heisenberg manifolds, Mich. Math. J. 33 (1986), 253-271. MR0837583 (87k:58275)

[GW3] - Continuous families of isospectral Riemannian manifolds which are not locally isometric, J. Differential Geometry 47 (1997), 504-529. MR.1617640 (99a:58159)

[Gt1] R. Gornet, Equivalence of quasi-regular representations of two- and three-step nilpotent Lie groups, J. Funct. Anal. 119 (1994), 121-137. MR.1255275 (95a:22008)

[Gt2] - The length spectrum and representation theory on two and three-step nilpotent Lie groups, Contemporary Mathematics: Geometry of the Spectrum (R. Brooks, C. S. Gordon, P. Perry, eds.), vol. 173, AMS, 1994, pp. 133-156. MR1298202 (95i:22015)

[Gt3] A new construction of isospectral Riemannian nilmanifolds with examples, Mich. Math. J. 43 (1996), 159-188. MR.1381605 (97b:58143)

[Gt4] - The marked length spectrum vs. the p-form spectrum of Riemannian nilmanifolds, Comment. Math. Helv. 71 (1996), 297-329. MR1396677 (97e:58222)

[Gt5] - Continuous families of Riemannian manifolds, isospectral on functions but not on 1-forms, J. Geom. An. 10 (2000), no. 2, 281-298. MR1766484 (2001i:58065)

[Gt6] - The wave invariants of Riemannian two-step nilmanifolds, in preparation (2005).

[GtM1] R. Gornet and M. Mast, The length spectrum on two-step nilmanifolds, Ann. Sci. de École Norm. Sup. 33 (2000), 181-209. MR.1755115|(2001d:58042)

[GtM2] — Length minimizing geodesics and the length spectrum of Riemannian two-step nilmanifolds, J. Geom. An. 13 (2003), 107-143. MR1967040 (2004e:58060)

[Gu] V. Guillemin, Wave-trace invariants, Duke Math. J. 83 (1996), no. 2, 287-352. MR1390650 (97f:58131)

[GuU] V. Guillemin and A. Uribe, On the de Haas-van Alphen effect, Asymptotic Analysis 6 (1993), 205-217. MR.1201193 (94f:58129)

[Kp] A. Kaplan, Riemannian nilmanifolds attached to Clifford modules, Geom. Dedicata 11 (1981), 127-136. MR0621376 (82h:22008)

[Kt] T. Kato, A Short Introduction to Perturbation Theory for Linear Operators, SpringerVerlag, 1982. MR0678094 (83m:47015)

[LP] K. B. Lee and K. Park, Smoothly closed geodesics in 2-step nilmanifolds, Indiana Univ. Math. J. 45 (1996), 1-14. MR1406681 (97h:53044)

[MR] Miatello and Rosetti, Length spectra and p-spectra of compact flat manifolds, J. Geom. Anal. 13 (2003), 631-657. MR2005157|(2005a:58053)

[O] He Ouyang, On isospectral rigidity of deformations on two-step nilmanifolds, Ph.D. Thesis, Washington University in St. Louis.

[P1] H. Pesce, Déformations isospectrales de certaines nilvariétés et finitude spectrale des variétés de Heisenberg, Ann. Sci. École Norm. Sup. (4) 25 (1992), 515-538. MR1191734 (94c:58214) 
[P2] Déformations L-isospectrales sur les nilvariétés de rang deux, C. R. Acad. Sci. Paris, Sér. I 315 (1992), 821-823. MR.1184910 (94b:58101)

[P3] _ Calcul du spectre d'une nilvariété de rang deux et applications, Trans. AMS. 339 (1993), 433-461. MR:1145731 (93k:58227)

[P4] Une formule de Poisson pour les variétes de Heisenberg, Duke Math. J. 73 (1994), no. 1, 79-95. MR.1257278 (95i:58183)

[R] M. S. Raghunathan, Discrete Subgroups of Lie Groups, Ergebnisse der Mathematik und ihrer Grenzgebeite, vol. 68, Springer-Verlag, 1972. MR0507234 (58:22394a)

[Sel] A. Selberg, Harmonic analysis and discontinuous groups in weakly symmetric Riemannian spaces with applications to Dirichlet series, J. Indian Math. Soc. (N.S.) 20 (1956), 47-87. MR0088511 (19:531g)

[St] R. Strichartz, A guide to distribution theory and Fourier transforms, CRC Press, 1994. MR1276724 (95f:42001)

[V] V. S. Varadarajan, Lie Groups, Lie Algebras, and their Representations, Graduate Texts in Mathematics, vol. 102, Springer-Verlag, 1984. MR.0746308 (85e:22001)

[Z1] S. Zelditch, The inverse spectral problem for surfaces of revolution, J. Differential Geom. 49 (1998), no. 2, 207-264. MR 1664907 (99k:58188)

[Z2] Spectral determination of analytic bi-axisymmetric plane domains, Geom. Funct. An. 10 (2000), no. 3, 628-677. MR.1779616 (2001k:58064)

Department of Mathematics, University of Texas at Arlington, Arlington, Texas 76019-0408

E-mail address: rgornet@uta.edu 